\title{
Curcumol inhibits malignant biological behaviors and TMZ-resistance of glioma cells via reducing long noncoding RNA FoxD2-As1 promoted EZH2 activation
}

\author{
Xuyang Lv \\ Zhejiang Chinese Medical University \\ Jiangchuan Sun \\ Zhejiang Chinese Medical University \\ Linfeng $\mathrm{Hu}$ \\ Zhejiang Chinese Medical University \\ Ying Qian \\ Zhejiang Chinese Medical University \\ Chunlei Fan \\ Zhejiang Chinese Medical University \\ Nan Tian ( $\sim 20111003 @ z c m u . e d u . c n$ ) \\ Zhejiang Chinese Medical University https://orcid.org/0000-0002-8929-1259
}

\section{Research}

Keywords: Curcumol, LncRNAs, Epigenetic modification, EZH2, Glioma, Herbal medicine

Posted Date: April 13th, 2021

DOI: https://doi.org/10.21203/rs.3.rs-415245/v1

License: (a) (i) This work is licensed under a Creative Commons Attribution 4.0 International License.

Read Full License 
1 Title: Curcumol inhibits malignant biological behaviors and TMZ-resistance of glioma cells via reducing long noncoding RNA FoxD2-As1 promoted EZH2 activation

3 Authors: Xuyang $\mathrm{Lv}^{1 \#}$, Jiangchuan Sun ${ }^{1 \#}$, Linfeng $\mathrm{Hu}^{1}$, Ying Qian ${ }^{1}$, Chunlei Fan ${ }^{1}$, Nan $\operatorname{Tian}^{1 *}$

$4 \quad$ Xuyang Lv and Jiangchuan Sun contributed equally to this work

5 Affiliation:

$6{ }^{1}$ Molecular medicine Institute, Life Science College, Zhejiang Chinese medical university,

7 Hangzhou, Zhejiang, China

\section{Corresponding author:}

9 Dr Nan Tian, Molecular medicine Institute, Life Science College, Zhejiang Chinese medical university. Binjiang District, Binwen road 548, Hangzhou, China. 310058 


\section{Abstract}

Background: Although curcumol has been shown to possess antitumor effects in several cancers, its effects on glioma are largely unknown. Recently, lncRNAs have been reported to play an oncogenic role through epigenetic modifications. Therefore, here, we investigated whether curcumol inhibited glioma progression by reducing FOXD2-AS1-mediated enhancer of zeste homolog 2 (EZH2) activation.

Methods: MTT, colony formation, flow cytometry, Transwell, and neurosphere formation assays were used to assess cell proliferation, cell cycle, apoptosis, the percentage of CD133+ cells, the migration and invasion abilities, and the self-renewal ability. qRT-PCR, western blotting, immunofluorescence, and immunohistochemical staining were used to detect mRNA and protein levels. Isobologram analysis and methylation-specific PCR were used to analyze the effects of curcumol on TMZ resistance in glioma cells. DNA pull-down and Chip assays were employed to explore the molecular mechanism underlying the functions of curcumol in glioma cells. Tumorigenicity was determined using a xenograft formation assay.

Results: Curcumol inhibited the proliferation, metastasis, self-renewal ability, and TMZ resistance of glioma cells in vitro and in vivo. FOXD2-AS1 was highly expressed in glioma cell lines, and its expression was suppressed by curcumol treatment in a dose- and time-dependent manner. The forced expression of FOXD2-AS1 abrogated the effect of curcumol on glioma cell proliferation, metastasis, self-renewal ability, and TMZ resistance. Moreover, the forced expression of FOXD2-AS1 reversed the inhibitory effect of curcumol on EZH2 activation.

Conclusions: We showed for the first time that curcumol is effective in inhibiting malignant biological behaviors and TMZ-resistance of glioma cells by suppressing FOXD2-AS1-mediated 
60

61

62

63 2

64 65 66

EZH2 activation on anti-oncogenes. Our findings offer the possibility of exploiting curcumol as a promising therapeutic agent for glioma treatment and may provide an option for the clinical application of this natural herbal medicine.

Keywords: Curcumol, LncRNAs, Epigenetic modification, EZH2, Glioma, Herbal medicine (1) 53 \\ 54}

5

7

58

59

61 62 63 


\section{Background}

Traditional Chinese medicine (TCM) has been practiced for more than 2,000 years [1]. It is widely accepted today and its popularity is growing internationally, such as in Japan and in the UK $[2,3]$. Herbal medicine is the most important part of TCM, as it provides curative treatments for a number of diseases and physiological conditions [4-7]. Curcumol, a guaiane-type sesquiterpenoid hemiketal extract from the roots of the herb Rhizoma Curcumae, has attracted significant interest recently due to its beneficial pharmacological activities. It possesses multiple therapeutic effects, such as antimicrobial, anti-fibrotic, antioxidant, anti-inflammatory, and anticancer activities with low cytotoxicity $[8,9]$. Recently, it has been reported that curcumol inhibits the growth of various types of cancer cells, including gastric adenocarcinoma, melanoma, hepatic cancer, and colorectal cancer cells [10-13]. Moreover, curcumol is lipophilic and readily crosses the blood-brain barrier $[14,15]$. Therefore, we wanted to explore whether curcumol has inhibitory effects on glioma.

Glioma is the most common form of brain malignancy and is characterized by multiple somatic mutations and an aberrant activation of inflammatory responses [16]. The standard treatment for glioma patients is postoperative radiotherapy and adjuvant temozolomide (TMZ)-based chemotherapy [17]. However, therapeutic resistance and tumor recurrence seriously affect their efficacy. Thus, the development of new therapeutic strategies against glioma is important.

Enhancer of zeste homolog 2 (EZH2), a member of the polycomb group $(\mathrm{PcG})$ gene family, is a critical subunit of polycomb repressive complex 2 (PRC2). PRC2 causes the methylation of histone 3 lysine 27 (H3K27me3) and closes the chromosome to epigenetically silence gene 
expression [18]. EZH2 plays a tumorigenic role through epigenetic activation of oncogenic signaling pathways [19]. Several studies have shown that reduced the expression of EZH2 in tumor cells can inhibit proliferation, migration, invasion, angiogenesis, and induce apoptosis [20-22]. In glioma, EZH2 has been verified to be overexpressed in tumor tissues and cell lines, and an elevated EZH2 expression is associated with the glioma grade and a poor prognosis in glioma patients [23, 24]. EZH2 also plays a critical role in glioma stem-like cell maintenance [25]. Recent studies have suggested that the long non-coding RNA FOXD2-AS1 recruits EZH2 to reduce the level of $\mathrm{H} 3 \mathrm{~K} 27 \mathrm{me} 3$ in the promoter regions of anti-oncogenes to epigenetically regulate the malignant progression of tumors [26-28]. FOXD2-AS1 is overexpressed in glioma and acts as an oncogene that promotes glioma malignancy and tumorigenesis $[29,30]$. Our previous results showed that curcumol treatment distinctly reduced the expression level of FOXD2-AS1 in glioma cells. Therefore, we investigated whether curcumol inhibited the development of glioma by reducing FOXD2-AS1-mediated and EZH2-induced H3K27me3 level in the promoter regions of anti-oncogenes. In this study, we tested this hypothesis to clarify the characteristics and molecular mechanisms of curcumol in glioma.

\section{Methods}

\section{Cell lines and materials}

Normal human astrocytes (NHAs) and glioma cell lines U251, A172 were purchased from Cell Resource Center of Shanghai and cultured in Dulbecco's Modified Eagle Medium (DMSO; Gibco, USA) supplemented with $10 \%$ fetal calf serum (FBS; Gibco, USA), 1\% penicillin, $1 \%$ streptomycin at $37^{\circ} \mathrm{C}$ in 5\% CO2. Curcumol and temozolomide (HY-N0104 and HY-17364, MCE,

USA) were dissolved in DMSO and diluted to appropriate concentrations in culture 
111

112

113

medium immediately before use.

\section{RNA isolation and qRT-PCR analysis}

Total RNA of cells and tissues was isolated by using TRIzol reagent (Invitrogen, USA) and converted to cDNA by HiFi-MMLV cDNA Kit (Beijing ComWin BiotechCo., Ltd. Beijing, China). QRT-PCR was conducted by using UltraSYBR Mixture (Beijing ComWin Biotech Co., Ltd. Beijing, China). The primer sequences were illustrated as follows: FOXD2-AS1, F: 5'-CTCACATCCGGCGGCT-3'， R: 5'-GGCTGTTCATGATATGTGCCA-3'; CDKN1B， F: 5'-GCCGCAACCAATGGATCTCCTC-3', R: 5'-AGTCGCAGAGCCGTGAGCAA-3'; EPHB3, F: 5'-TGGGTAACATCTGAGTTGGCG-3', R: 5'-TGGTATGTGCGGATGGGATTC-3'; p21, F: 5'TGTGATGAAAGACGGCACAC-3'， R: 5'-CTTCCTTTGGGTATTGTTTGG-3'; EZH2， F： 5'GGACCACAGTGTTACCAGCAT-3'，R： 5'-GTGGGGTCTTTATCCGCTCAG-3'; $\beta$-actin，F: 5'-GGCACCACACCTTCTACAAT-3', R: 5'-GTGGTGGTGAAGCTGTAGCC-3'. All the primers were synthesized by Nanjing GenScript Co. Ltd., China. The fold-change of each gene was calculated using the $2^{-\Delta \Delta \mathrm{Ct}}$ method.

\section{Genome DNA extraction and Methylation-specific (MS) PCR}

For DNA extraction from cells, Takara MiniBEST Universal Genomic DNA Kit (Takara, Shiga, Japan) was used, following the manufacturer's directions. After bisulfite treatment by a DNA Methylation Kit (CoWin Biosciences, Beijing, China), $4 \mu \mathrm{L}$ of the purified DNA was used to perform MS-PCR. Primers for the methylated MGMT were F: 5'-TTTCGACGTTCGTAGGTTTTCGC-3', R: 5'-GCACTCTTCCGAAAACGAAACG-3'; for the un-methylated MGMT were F: 5'-TTTGTGTTTTGATGTTTGTAGGTTTTTGT-3', R: 5'-AACTCCACACTCTTCCAAAAACAAAACA-3'. The PCR products were directly loaded 
133

onto $2.5 \%$ agarose gels, stained with ethidium bromide, and visualized with the aid of ultraviolet light. The density of each band was quantified using imaging analysis and the relative band density values were calculated as the ratio of methylated MGMT to that of methylated plus un-methylated MGMT. The MS-PCR was performed in a Mastercycler Gradient (Eppendorf) under the following conditions: $95^{\circ} \mathrm{C}$ for $10 \mathrm{~min}$; 35 cycles of $95^{\circ} \mathrm{C}$ for $30 \mathrm{sec}$, the annealing temperature $60^{\circ} \mathrm{C}$ for $30 \mathrm{sec}$, and $72^{\circ} \mathrm{C}$ for $30 \mathrm{sec}$; and a final extension of $10 \mathrm{~min}$ at $72^{\circ} \mathrm{C}$.

\section{Cell viability}

Glioma cells were placed on 96 -well plates at a density of $5 \times 10^{3} /$ well and after overnight incubation treatment with curcumol for 48 hours. MTT solution $(5 \mathrm{mg} / \mathrm{mL})$ was added to each well and the plate was incubated for $4 \mathrm{~h}$ at $37^{\circ} \mathrm{C}$. After removal of the medium, formazan crystals were dissolved in $150 \mu \mathrm{L}$ of DMSO. The absorbance value was measured at $550 \mathrm{~nm}$ using a SpectraMaxM3 microplate reader (MolecularDevices, Sunnyvale, CA, USA).

\section{Migration and invasion assays}

Glioma cells were suspended in DMEM without FBS and seeded into the upper chambers of the transwell inserts (Corning, Corning, NY, USA). Afterwards, the DMEM added with 20\% FBS was added to the lower chambers. The invasion assay was performed in the same way as the migration assay except that the membrane was pre-treated with matrigel (BD Biosciences, Bedford, MA, USA).The migrated or invaded cells through the member were fixed with $4 \%$ paraformaldehyde and stained with $0.1 \%$ crystal violet. The stained cells were counted under microscope (ECLIPSE Ti-S, Nikon, Japan).

\section{Colony formation assay}

For colony formation assay, treated and untreated glioma cells were placed at a density of 500 
155

156

157

cells per well into 6 -well plates and then cultured at $37^{\circ} \mathrm{C}$ for 2 weeks, stained with $5 \%$ crystal violet. Finally, colonies were photographed, and the number of colonies was counted manually.

\section{Annexin V-FITC/PI apoptosis assay}

Annexin V-FITC/PI staining Kit (NanJing KeyGen Biotech Co., Ltd., Nanjing, China) was utilized to detect the cell apoptotic rates. The treated and untreated cells were collected and resuspended in the binding buffer. After incubating in the dark for $15 \mathrm{~min}$, the cells were analyzed using a Guava easyCyte 5 Flow Cytometer (EMD Millipore, Bellerica, MA, USA).

\section{Neurosphere formation}

Glioma cells $\left(3 \times 10^{4} /\right.$ well $)$ were plated into ultra-low attached 6-well plates (Corning Costar Inc, Corning, NY, USA) and cultured in serum-free DMEM/F12 media (Gibco, USA) with 2\% B27 supplement (Thermo Fisher Scientific, USA), 20 ng/mL EGF, and 10 ng/mL bFGF (PeproTech, Rocky Hill, NJ, USA). After 4-5 days, equal fresh media was added. Cells were incubated for 2 weeks, and spheres with diameter $\geq 50 \mu \mathrm{m}$ were counted.

\section{Flow cytometric analysis (FCM)}

Glioma cells $\left(1 \times 10^{6} /\right.$ well $)$ were washed twice with PBS supplemented with $0.5 \%$ BSA and $2 \mathrm{mM}$ EDTA and incubated in diluted CD133-PE antibody solution (Invitrogen, USA) for 15 min. After washing the cells twice, the percentage of CD133+ cells was analyzed by Guava easyCyte 5 Flow Cytometer (EMD Millipore, Bellerica, MA, USA).

\section{Immunofluorescence staining}

For immunofluorescence staining neurospheres, the cells were fixed with $4 \%$ paraformaldehyde and incubated with antibodies against CD133 (18470-1-AP, Proteintech gruop, USA) and Nanog (ab62734, Abcam, USA) overnight at $4^{\circ} \mathrm{C}$. Then, SABC (rabbit IgG)-FITC/ 
177

SABC (mouse IgG)-Cy3 Kit (Boster Bioengineering, Wuhan, China) was used. Nuclei were stained with 4'6-diamidino-2-phenylindole (DAPI). Fluorescence images were captured with fluorescent microscope (ECLIPSE Ti-S, Nikon, Japan).

\section{Western blotting}

Tissues and cells were lysed with RIPA (Boster Bioengineering, Wuhan, China) containing $1 \mathrm{mM}$ phenylmethanesulfonyl fluoride (PMSF; Roche Molecular Biochemicals, Indianapolis, IN, USA). Protein samples were separated by $10 \%-12 \%$ SDS PAGE. Western blotting was performed based on a previous study [31]. The primary antibody to $\beta$-actin was purchased from Hua Bio (HuaAn Biotechnology Co., Ltd., Hangzhou, China), antibody to EphB3 was purchased from Invitrogen (Invitrogen, USA), the antibodies to MGMT, Nestin, SOX2, p21, CDKN1B were purchased from (Proteintech Group, Chicago, IL, USA), and the antibodies to EZH2 and Histone H3 (H3K27me3) were purchased from Abcam (Abcam, Cambridge, USA).

\section{Generation of TMZ-resistant glioma cells}

To develop a TMZ-resistant glioma cell line, U251 cells were cultured in media containing increasing concentrations of TMZ. Briefly, U251 cells were seeded in culture flasks at a density of $4 \sim 5 \times 10^{5}$ cells $/ \mathrm{mL}$ and allowed to grow. After $24 \mathrm{~h}$ incubation, low dose TMZ was added, and the cells were incubated for another $24 \mathrm{~h}$. Then, the cells were washed 3 times with PBS and the medium was changed to drug-free medium. The cells were incubated and allowed to grow until confluent. Then, the cells were subcultured and re-exposed to higher dose of TMZ. After successful development, TMZ-resistant U251 cells were maintained in $2 \mu \mathrm{g} / \mathrm{mL}$ TMZ.

\section{Isobologram analysis}

For combination assay, $\mathrm{IC}_{50}$ was calculated from two sets of concentration response graphs, the 
199

200

201

202

203

204

205

combination index $(\mathrm{CI})$ was calculated according to the following equation [32]: $\mathrm{CI}=(d 1 / D x 1)+$ (d2/Dx2). $D x 1$ is the concentration of drug 1 (TMZ) required to produce x percentage effect alone, $d 1$ is the concentration of drug 1 required to produce the same $\mathrm{x}$ percentage effect in combination with $d 2 . D x 2$ is the concentration of drug 2 (curcumol) required to produce x percentage effect alone, and $d 2$ is the concentration of drug 2 required to produce the same x percentage effect in combination with $d 1$. The $\mathrm{CI}$ values were defined as follows: $<1$, synergism; $=1$, additive; and $>1$, antagonism.

\section{Plasmids' construction and stable transfection}

The FOXD2-AS1 sequence was synthesized from GenScript Co. Ltd., (GenScript, Nanjing, China) and subcloned into the pcDNA3.0 vector. For establishing the FoxD2-As1 stable overexpression glioma cell lines, pcDNA-FoxD2-As1 or empty vector was transfected into cells using Lipofectamin 2000. After $72 \mathrm{~h}$ incubation, G418 was then added to the transfected cells for generating cells stably overexpressing FoxD2-As1. The transfection effect was tested by qRT-PCR.

\section{DNA pull down assay}

Biotinylated DNA probes corresponding to the EphB3, p21, and CDKN1B promoter was prepared with PCR using glioma cell genome DNA as the template. The oligonucleotides used, corresponding to the EphB3 promoter was F: 5'-ACCCGCAGGTACTACAGTCT-3', R: 5'-CACCGCGATGTATCCTGTGA-3'; to the p21 promoter was 5'-GAGGTCAGCTGCGTtAGAGG-3'， R: 5'-TGCAGAGGATGGATTGTTCA-3'; to the CDKN1B promoter was 5'-AAGTGCCGCGTCTACTCCTG-3', R: 5'-TGGAGGCAGGgCAATGGT-3'. The biotinylated DNA probe $(1 \mu \mathrm{g}) 30 \mu \mathrm{L}$ streptavidin 
221

222

223

224

225

agarose beads (Sigma-Aldrich) were mixed and incubated in buffer (10 mM Tris- $\mathrm{HCl}(\mathrm{pH} 8.0)$, $0.5 \mathrm{mM}$ EDTA, $1 \mathrm{M} \mathrm{NaCl}$ ) at $4{ }^{\circ} \mathrm{C}$ overnight. Nuclear extracts were prepared from glioma cells using the Nuclear Extraction Kit (Takara, Japan). $400 \mu \mathrm{g}$ of cell nuclear protein extract was incubated with DNA-coupled magnetic beads at $4{ }^{\circ} \mathrm{C}$ for $1 \mathrm{~h}$, and then the beads were washed three times with cell lysis buffer and once with PBS. After boiled for 5 min in $2 \times$ SDS-loading buffer, the samples were analyzed by western blotting using EZH2 and H3K27me3 antibodies.

\section{Chromatin immunoprecipitation (Chip) assay}

The ChiP-IT Express Enzymatic Kit (Active Motif, Carlsbad, USA) was used for Chip assay.

Glioma cells were fixed with formaldehyde, harvested and chromatin was enzymatically sheared. Chromatin immune precipitates were isolated on protein G magnetic beads using $2 \mu \mathrm{g}$ of IgG, EZH2, or H3K27me3 antibodies. After washing, the Chips were eluted, reverse cross-linked, and subjected to qRT-PCR. Chips were normalized to input DNA and the IgG control.

\section{Mouse tumor xenografts}

BALB/c nude mice (male, 5-week-old, 16-20 g) were purchased from Shanghai Slack Laboratory Animal Co. Ltd. The experiment was approved by the Institutional Animal Care and Use Committee of Zhejiang Chinese medical university laboratory animal research center, and animal studies were performed in compliance with the Guidelines for Care and Use of Laboratory Animals of National Institutes of Health and the Basel Declaration. 251/TMZ cells $\left(1 \times 10^{7}\right)$ were injected into each mouse. After 7 days, the mice were randomly assigned to three groups $(n=5)$, and either treated with or without DMSO, curcumol $(20 \mathrm{mg} / \mathrm{kg})$ by intraperitoneal injections every 3 days, respectively. Tumor volume was calculated using the formula $\mathrm{V}=\mathrm{W}^{2} \times \mathrm{L} \times 0.5$. The mice were sacrificed and tumors were harvested for the follow-up experiments. 


\section{Immunohistochemistry (IHC)}

The dissected tumors sections $(5 \mu \mathrm{m})$ mounted on the glass slides were deparaffinized and rehydrated. Subsequently, the specimens were washed in 0.01 M PBS, treated with $3 \%$ hydrogen peroxide, and washed in $0.01 \mathrm{M}$ PBS again. Blocking was performed with $3 \% \mathrm{BSA}$ in $0.01 \mathrm{M}$ PBS. Then the sections were incubated with antibodies for proliferation marker Ki-67, CD133, MMP2 and MGMT, followed by incubation with secondary antibodies. After the cell nuclei were labeled with hematoxylin, images were captured using a microscope (XSP-C204, Chongqing Optical Instrument Co., Ltd.).

\section{Statistical analysis}

Data were presented as mean \pm standard deviation (SD). The statistical analysis was performed using Student's $t$-test (for comparison in two groups) or one-way analysis of variance (ANOVA; for comparison in multiple groups). Turkey's post hoc test was performed after ANOVA. Statistical significance was determined at $\mathrm{p}<0.05$.

\section{Results}

\section{Curcumol inhibited proliferation and metastasis, and promoted apoptosis in glioma cells}

To determine the activity of curcumol in glioma cells, we first tested whether curcumol had an inhibitory effect on the proliferation of glioma cells. Using an MTT assay, we observed that curcumol significantly decreased the viability of U251 and A172 cells, except at a dose of 10 $\mu \mathrm{g} / \mathrm{mL}$ (Fig. 1A). Curcumol showed a dose-dependent cytotoxic effect against A172 cells (Fig. 1A). Curcumol was also observed to inhibit the migratory and invasive abilities of these two glioma cell lines, as shown in Fig. 1B and 1C, in which cellular treatment with curcumol (20 and 
$40 \mu \mathrm{g} / \mathrm{mL}$, for $48 \mathrm{~h}$ ) clearly inhibited cell migration and invasion. Furthermore, to determine whether the inhibitory effect of curcumol on glioma cells is associated with cell apoptosis, we analyzed the apoptosis-inducing effect of curcumol in U251 and A172 cells. The results of Annexin V-FITC/PI fluorescence staining assay showed that curcumol induced glioma cell apoptosis in a dose-dependent manner at $48 \mathrm{~h}$.

\section{Curcumol inhibited the self-renewal ability of glioma cells}

We enriched a CD133+ "stem-like" neurosphere from U251 and A172 glioma cells by growing them in stem-like conditions (Fig. 2A). A neurosphere formation assay was used to assess the impact of curcumol on the self-renewal ability of glioma cells. As shown in Fig. 2B, curcumol (40 $\mu \mathrm{g} / \mathrm{mL}$ ) decreased the number of spheres relative to the control group, and curcumol-treated spheres were significantly smaller than control spheres. Immunofluorescence of the putative glioma stem cell markers CD133 and Nanog also showed that curcumol treatment significantly decreased CD133 and Nanog protein levels when the glioma cell lines were grown as stem-like neurospheres (Fig. 2A). To estimate the percentage of CD133+ "stem-like" cells in the cancer cell population after curcumol treatment, the expression of CD133 was evaluated using FCM. Our results showed that curcumol treatment decreased the percentage of CD133+ cells in both U251 and A172 cell lines (Fig. 2C). We further assessed the stemness of glioma cells by measuring a panel of stem cell marker genes using western blotting analysis and found a significantly reduced expression of CD133, Nestin, Nanog, and SOX-2 after curcumol treatment (Fig.2D).

\section{Curcumol increased the sensitivity of glioma cells to TMZ}

We generated a chemoresistant cell line against TMZ by culturing U251 cells in media containing increasing concentrations of TMZ, and then maintained them at $2 \mu \mathrm{g} / \mathrm{mL}$ TMZ (Fig. 3A). To 
confirm the acquired chemoresistance of these cells, we analyzed their viability at various TMZ concentrations compared to their parental counterparts. We found that U251/TMZ cells were able to proliferate at higher concentrations of TMZ than the parental cell line (Fig. 3B). Next, we evaluated the effect of curcumol on TMZ sensitivity in U251/TMZ cells by determining the changes in cellular proliferation after treatment with curcumol or TMZ alone, or in combination. U251/TMZ cells appeared to show resistance to low-dose TMZ, but not to curcumol. However, the combined treatment further enhanced cellular cytotoxicity (Fig. 3C). Isobologram analysis indicated that TMZ and curcumol synergistically enhanced the cytotoxicity against U251/TMZ cells $(\mathrm{CI}=0.82$ for $16.25 \mu \mathrm{g} / \mathrm{mL}$ curcumol combined with $62.38 \mu \mathrm{g} / \mathrm{mL} \mathrm{TMZ}$; $\mathrm{CI}=0.83$ for 32.50 $\mu \mathrm{g} / \mathrm{mL}$ curcumol combined with $50.87 \mu \mathrm{g} / \mathrm{mL} \mathrm{TMZ}$; $\mathrm{CI}=0.90$ for $65 \mu \mathrm{g} / \mathrm{mL}$ curcumol combined with $32.23 \mu \mathrm{g} / \mathrm{mL}$ TMZ; Fig.3C). Moreover, we performed a colony formation assay to determine the effects of curcumol and/or TMZ on the proliferation and survival of U251/TMZ cells. As shown in Fig. 3D, TMZ treatment alone did not effectively reduce the number of colonies in $\mathrm{U} 251 / \mathrm{TMZ}$ cells at a lower dose $(5 \mu \mathrm{g} / \mathrm{mL})$ and slightly reduced the colony formation rate $(10.21 \%)$ at a dose of $10 \mu \mathrm{g} / \mathrm{mL}$. Curcumol treatment alone effectively suppressed the colony formation of U251/TMZ cells, and the combination of the two compounds further inhibited the clonogenic capacity.

Recently, O6-methylguanine-DNA methyltransferase (MGMT) promoter methylation has been implicated in drug resistance of TMZ against glioma. A high promoter methylation status which means low MGMT activity predicts good response to TMZ chemotherapy and results in a longer survival period in glioma patients [31]. Therefore, we confirmed the methylation status of the MGMT promoter in U251/TMZ cells treated with curcumol and/or TMZ using MS-PCR. As 
shown in Fig. 3E, curcumol treatment alone significantly increased the ratio of promoter DNA methylation, and the combination of curcumol and TMZ further increased this ratio. However, TMZ treatment alone did not induce the hypermethylation of the MGMT promoter region. Western blotting analysis showed that curcumol, alone or in combination with TMZ, suppressed the expression of MGMT (Fig. 3E, bottom panel). Taken together, these results suggest that curcumol increases the sensitivity of glioma cells to TMZ.

FOXD2-AS1 expression was up-regulated in glioma cells and curcumol treatment down-regulated FOXD2-AS1

To elucidate the underlying mechanism of curcumol in glioma, we explored the role of FOXD2-AS1 in this process. We found that there was a marked upregulation of FOXD2-AS1 in glioma cells compared to NHAs (Fig. 4A). Next, we analyzed FOXD2-AS1 expression in glioma cells after treatment with curcumol, for 24,48 , and $72 \mathrm{~h}$. Curcumol treatment decreased the expression of FOXD2-AS1 in glioma cells in a dose- and time-dependent manner (Fig. 4B).

FOXD2-AS1 over-expression abrogated the effect of curcumol on the proliferation, metastasis, and apoptosis of glioma cells

To further evaluate the function of FOXD2-AS1 in the inhibitory effect of curcumol on glioma cells, U251 and A172 cells were transfected with pcDNA3.1-FOXD2-AS1 or empty vector, followed by treatment with curcumol for $48 \mathrm{~h}$ (Fig. 5A). As shown by the MTT assay results, in curcumol-treated U251 and A172 cells, the overexpression of FOXD2-AS1 remarkably promoted cell viability compared to the control and empty vector-transfected cells (Fig. 5B). Moreover, Transwell migration and invasion assays showed that the overexpression of FOXD2-AS1 abrogated the curcumol-induced inhibition of cell metastasis (Fig. 5C and D). The overexpression 
331

of FOXD2-AS1 in curcumol-treated glioma cells prominently reduced the apoptotic rate compared to the control and empty vector-transfected groups (Fig. 5E).

FOXD2-AS1 over-expression abrogated the effect of curcumol on the self-renewal ability of glioma cells

We continued to determine whether FOXD2-AS1 overexpression abolished the inhibitory effect of curcumol on the self-renewal ability of glioma cells. As shown in Fig. 6A and B, the forced expression of FOXD2-AS1 significantly promoted neurosphere formation and the expression of CD133 and Nanog in U251 and A172 cells, which grow in stem-like conditions in the presence of curcumol. The upregulation of FOXD2-AS1 also increased the curcumol-mediated reduction of the CD133+ cell percentage in both U251 and A172 cell lines (Fig.6C). Moreover, western blotting analysis results showed that a forced expression of FOXD2-AS1 reversed the curcumol-mediated decrease in CD133, Nestin, Nanog, and SOX-2 protein expression (Fig. 6D). These results demonstrated that the overexpression of FOXD2-AS1 abrogated the effect of curcumol on the self-renewal ability of glioma cells.

FOXD2-AS1 over-expression abrogated the effect of curcumol on TMZ-resistance of glioma cells

We further investigated the function of FOXD2-AS1 in the effect of curcumol on TMZ resistance in glioma cells. As shown in Fig. 7A and B, FOXD2-AS1 overexpression reversed the cytotoxic effects of curcumol and TMZ on U251/TMZ cells and increased the colony numbers, which were decreased by curcumol and TMZ treatment. Furthermore, compared with the control and empty vector cells, FOXD2-AS1 overexpression significantly reduced the ratio of methylated MGMT promoter DNA and promoted MGMT protein expression in U251/TMZ cells (Fig. 7C). The above 
353

results showed that FOXD2-AS1 upregulation abrogated the effect of curcumol on TMZ resistance in glioma cells.

\section{Curcumol inhibited the activation of EZH2 through the downregulation of FOXD2-AS1}

Recent research has demonstrated that FOXD2-AS1 acts as a tumor inducer by promoting the recruitment of EZH2 to tumor suppressor gene promoters and increasing H3K27me3 modification. We hypothesized that curcumol modulated the activation of EZH2 through FOXD2-AS1 to exert antitumor activity. Thus, we first detected the activation of EZH2 in glioma cells with or without curcumol treatment. There is evidence that FOXD2-AS1 epigenetically silences EphB3 [26], $C D K N 1 B$ [27], and p21 [28] through EZH2; therefore, we performed DNA pull-down and Chip assays to determine the effect of curcumol on the recruitment of EZH2 to EphB3, CDKN1B, and $p 21$ promoters. As shown in Fig. $8 \mathrm{~A}$ and $\mathrm{B}, 40 \mu \mathrm{g} / \mathrm{mL}$ curcumol treatment decreased the binding ability of EZH2 to EphB3, p21, and $C D K N 1 B$ promoters in glioma cells. Meanwhile, curcumol inhibited $\mathrm{H} 3 \mathrm{~K} 27 \mathrm{me} 3$ modification in the promoter regions of $E p h B 3, p 21$, and $C D K N 1 B$. As expected, curcumol significantly increased the expression levels of EphB3, p21, and CDKN1B in glioma cells (Fig. 8C). These results suggest that curcumol inhibits the activation of EZH2.

To evaluate whether FOXD2-AS1 is involved in curcumol-reduced EZH2 activity, we overexpressed FOXD2-AS1 in glioma cells. DNA pull-down and Chip assays results showed that the overexpression of FOXD2-AS1 significantly reversed the inhibitory effect of curcumol on the binding ability of EZH2 and $\mathrm{H} 3 \mathrm{~K} 27 \mathrm{me} 3$ modification in the promoter regions of EphB3, p21, and $C D K N 1 B$ (Fig.8A and B). FOXD2-AS1 overexpression reversed the promotional effect of curcumol on the expression levels of EphB3, p21, and CDKN1B (Fig. 8C). Moreover, we found that curcumol inhibited the expression of EZH2 (Fig. 8D). 
375

376

377

378

379

380

381

382

383

384

\section{Curcumol inhibited malignant biological behaviors and TMZ-resistance of glioma cells in}

vivo

Further, for the in vivo studies, U251/TMZ cells were subcutaneously injected into nude mice, and then these were treated with intraperitoneal injections of curcumol or DMSO for 12 days. Curcumol treatment significantly suppressed U251/TMZ cell-derived tumor growth based on the tumor volume and weight (Fig. 9A). Then, tumor biopsies from the three groups were collected and cultured to examine the sphere-forming ability of the cells and their tolerance to TMZ. As shown in Fig. 9B, under serum-deprived conditions, the tumor samples from the curcumol group showed a significantly decreased sphere-forming ability compared to the control and DMSO groups. Moreover, the percentage of CD133+ cells in tumor samples from the curcumol group was significantly lower than that in the control and DMSO groups (Fig.9C). After treatment with a concentration series of TMZ for $48 \mathrm{~h}$, the cells from the tumor samples of the control and DMSO groups showed a high tolerance to TMZ; however, the cells from the tumor samples of the curcumol group showed a low tolerance to TMZ (Fig.9D). We then assessed the expression of CD133, MMP2, and MGMT in the tumors of each group using western blotting and IHC. The results showed that curcumol treatment markedly reduced the protein levels of CD133, MMP2, and MGMT (Fig. 9E and F). Meanwhile, the level of the proliferation marker protein Ki-67 was much lower in curcumol-treated tumors (Fig. 9F). These findings demonstrated that curcumol inhibited the proliferation, self-renewal ability, metastasis, and TMZ resistance of glioma cells in vivo.

\section{Discussion}



the survival of glioma patients. Hence, it is time to adopt novel personalized treatment programs, such as targeted therapy, immunotherapy, traditional Chinese medicine therapy, and other methods. Chinese herbal medicine is an important part of complementary and alternative medicine, and its potential in the treatment of glioma has been gradually demonstrated. In China, herbal medicine combined with radiotherapy and/or chemotherapy has achieved good results in improving the adverse reactions and the quality of life of glioma patients, preventing postoperative recurrence, and prolonging survival [33-36]. Moreover, several preclinical studies have demonstrated an inhibitory effect of natural compounds extracted from herbal medicines on glioma cell growth [37-39]. In the present study, we focused on curcumol, a well-known herbal medicine and healthfood supplement in China. Our study revealed that curcumol treatment inhibited the proliferation and invasion of glioma cells, while promoting cell apoptosis. Using in vitro and in vivo experiments, we also found that curcumol treatment decreased the percentage of CD133+ glioma cells and reduced the sphere-formation and protein levels of stem cell markers in glioma cells, indicating the inhibitory effects of curcumol on glioma self-renewal ability.

$M G M T$ promoter methylation is the key mechanism of $M G M T$ gene silencing and predicts a favorable TMZ chemotherapy outcome in glioma patients. The regents increasing the rate of MGMT promoter methylation have potiential to be a sensitizer in TMZ treatment. In this study, we resistance in glioma cells. Our results showed that curcumol synergized with TMZ to reduce the viability and colony formation ability of U251/TMZ cells. Synergistically with TMZ, curcumol also showed a promotional effect on the ratio of $M G M T$ promoter methylation and an inhibitory 
effect on MGMT protein levels. In addition, curcumol treatment also showed a good inhibitory effect on the growth of 251/TMZ cell xenografts. These data suggest the potential of curcumol to sensitize glioma cells to TMZ.

The inhibitory effects of curcumol on different cancer cells involve different molecular mechanisms. In colorectal cancer cells, curcumol promotes cell death through the IGF-1R and p38 MAPK pathways [40]; in melanoma, curcumol inhibited cell proliferation and metastasis via the miR-152-3p/PI3K/AKT and ERK/NF-kappaB signaling pathways [41], and the inhibitory effects of curcumol on gastric adenocarcinoma cells via downregulation of isocitrate dehydrogenase 1 [42]. Here, we found that FOXD2-AS1 might play a role in the effects of curcumol on glioma cells. An enforced expression of FOXD2-AS1 attenuated the curcumol-induced reduction in glioma cell proliferation, metastasis, self-renewal ability, and TMZ resistance. FOXD2-AS1, located on chromosome1p33, with a transcript with a length of 2,527 nucleotides, is reported to be highly expressed in glioma tissues $[29,30]$. The current study demonstrated that curcumol decreased the expression of FOXD2-AS1 in glioma cells in a dose- and time-dependent manner, indicating that FOXD2-AS1might be a molecular target of curcumol treatment in glioma.

More recently, lncRNAs were found to act as a scaffold for various epigenetic proteins and influence the epigenetic state of chromatin to regulate tumor progression [43]. Shu et al. demonstrated that FOXD2-AS1 promoted gastric cancer carcinogenesis by epigenetically silencing EphB3 through EZH2 [26]. Xu et al. revealed that FOXD2-AS1 plays an oncogenic role in hepatocellular carcinoma through epigenetic silencing of CDKN1B via EZH2 [27]. EZH2 has also been investigated as a cancer therapeutic target, with numerous studies suggesting its potential therapeutic benefits against glioma. We further investigated whether curcumol inhibited 
the malignant behavior of glioma cells by reducing FOXD2-AS1-induced EZH2 activity. We

441 found that curcumol treatment decreased the binding ability of EZH2 to the promoter of

442 downstream effectors (EphB3 and CDKN1B) and the H3K27me3 modification in the

443 promoter regions of effectors, and increased the expression level of effectors in glioma.

444 Furthermore, an enforced expression of FOXD2-AS1 reversed the effects of curcumol on EZH2

445 downstream effectors. In addition, we found that curcumol inhibited the expression of EZH2,

446 which is consistent with the results of $\mathrm{Li}$ et al. [44]. Hence, we constructed a direct interaction

447 between curcumol treatment and FOXD2-AS1-induced EZH2 activity in glioma cells.

\section{$448 \quad$ Conclusions}

In summary, our data demonstrated that curcumol is effective in inhibiting the proliferation,

metastasis, self-renewal ability, and TMZ resistance of glioma cells in vitro and in vivo. Moreover,

might be a molecular target of curcumol treatment and that curcumol performs its functions in the potential of curcumol as a promising therapeutic agent for glioma and may provide an option therapeutic dose of curcumol, which can eliminate glioma cells while minimizing damage to normal cells, must be conducted.

\section{List of abbreviations}

Traditional Chinese medicine (TCM), enhancer of zeste homolog 2 (EZH2), polycomb group

(PcG), polycomb repressive complex 2 (PRC2), methylation of histone 3 lysine 27 (H3K27me3), 
462 (DMSO), fetal calf serum (FBS), 4'6-diamidino-2-phenylindole (DAPI), phenylmethanesulfonyl 463 fluoride (PMSF).

\section{Acknowledgements}

465 Thanks for all participants involved in this research.

Authors' contributions

NT, CLF designed this study. LFH and YQ contributed to analysis and interpretation of data, and assisted in the preparation of the manuscript. NT reviewed and edited the manuscript. XYL, JCS performed experiments and wrote the initial draft of the manuscript.

\section{Funding}

This study was supported in part by research grants from the Natural Science Foundation of

472 Zhejiang Province (No. LY20H280005), National Natural Science Foundation of China

473 (81602624), Science and Technology Innovation Activity Program for Undergraduates

474 in Zhejiang Province-Xinmiao Talent Program (2020R410027).

\section{Availability of data and materials}

476 The datasets used and analyzed during the current study are available from the corresponding

477 author on reasonable request.

478 Ethics approval and consent to participate

479 The animal procedures were authorized by the Institutional Animal Care and Use Committee of 480 Zhejiang Chinese medical university laboratory animal research center in compliance with the 481 NIH Guide for Care and Use of Laboratory Animals.

\section{Consent for publication}

483 Not applicable. 


\section{Competing interests}

485

486

487

488

489

490

491

492

493

494

495

496

497

498

499

500

501

502

503

504

505

The authors declare no conflicts of interest.

\section{References}

1. Wang D, Calabrese EJ, Lian B, Lin Z. Hormesis as a mechanistic approach to understanding herbal treatments in traditional Chinese medicine. Pharmacol Ther. 2018;184:42-50. doi: 10.1016/j.pharmthera.2017.10.013.

2. MotooY, Seki T, Tsutani K. Traditional Japanese medicine, Kampo: Its history and current status. Chin J Integr Med. 2011;17(2): 85-87. doi: 10.1007/s11655-011-0653-y.

3. Scheid V, Tuffrey V, Weijburg T, Bovey M, Ward T. Chinese medicine treatment for menopausal symptoms in the UK health service: Is a clinical trial warranted? Maturitas. 2015;80(2):179-186. doi: 10.1016/j.maturitas.2014.11.006.

4. Yang Y, Islam MS, Wang J, Li Y, Chen X. Traditional Chinese Medicine in the Treatment of Patients Infected with 2019-New Coronavirus (SARS-CoV-2): A Review and Perspective. Int J Biol Sci. 2020;16(10):1708-1717. doi: 10.7150/ijbs.45538.

5. Singh A, Zhao K. Treatment of Insomnia With Traditional Chinese Herbal Medicine. Int Rev Neurobiol. 2017;135:97-115. doi: 10.1016/bs.irn.2017.02.006.

6. Yang X, Liu N, Li X, Yang Y, Wang X, Li L, Jiang L, Gao Y, Tang H, Tang Y, Xing Y, Shang H. A Review on the Effect of Traditional Chinese Medicine Against Anthracycline-Induced Cardiac Toxicity. Front Pharmacol. 2018;9:444. doi: 10.3389/fphar.2018.00444.

7. Wang J, Qi F, Wang Z, Zhang Z, Pan N, Huai L, Qu S, Zhao L. A review of traditional Chinese medicine for treatment of glioblastoma. Biosci Trends. 
8. Yin G, Cheng X, Tao W, Dong Y, Bian Y, Zang W, Tang D. Comparative analysis of multiple representative components in the herb pair Astragali Radix-Curcumae Rhizoma and its single herbs by UPLC-QQQ-MS. J Pharm Biomed Anal. 2018;148:224-229. doi: 10.1016/j.jpba.2017.09.015.

9. Wei W, Rasul A, Sadiqa A, Sarfraz I, Hussain G, Nageen B, Liu X, Watanabe N, Selamoglu Z, Ali M, Li X, Li J. Curcumol: From Plant Roots to Cancer Roots. Int J Biol Sci.

10. Huang X, Qian J, Li L, Zhang X, Wei G, Lv J, Qin F, Yu J, Xiao Y, Gong Z, Huo J. Curcumol improves cisplatin sensitivity of human gastric cancer cells through inhibiting PI3K/AKT pathway. Drug DevRes. 2020;81(8):1019-1025. doi: 10.1002/ddr.21719.

11. Ning N, Liu S, Liu X, Tian Z, Jiang Y, Yu N, Tan B, Feng H, Feng X, Zou L.

12. Zuo H, Jin Y, Wang Z, Li M, Zhang Z, Wang J, Xing Y, Ri MH, Jin C, Xu G, Piao L, Ma J, Jin X. Curcumol inhibits the expression of programmed cell death-ligand 1 through crosstalk Curcumol inhibits colorectal cancer proliferation by targeting miR-21 and modulated PTEN/PI3K/Akt pathways. Life Sci. 2019;221:354-361. doi: 10.1016/j.1fs.2019.02.049. 
14. Zhang R, Wang BJ, Zhao HL, Wei CM, Yuan GY, Guo RC. Tissue distribution of curcumol in rats after intravenous injection of zedoary turmeric oil fat emulsion. Asian J Pharmacodynamics Pharmacokinetics. 2009;9(1):51-57.

15. Liu Y, Fan H, Ding J, Huang C, Deng S, Zhu T, Xu T, Ge W, Li W, Li F. Curcumol allosterically modulates GABA(A) receptors in a manner distinct from benzodiazepines. Scientific reports. 2017;7(1):483-507. doi: 10.1038/srep46654.

16. Lapointe S, Perry A, Butowski NA. Primary brain tumours in adults. Lancet. 2018;392 (10145):432-446. doi: 10.1016/S0140-6736(18)30990-5.

17. Chua J, Nafziger E, Leung D. Evidence-Based Practice: Temozolomide Beyond Glioblastoma. Curr Oncol Rep. 2019;21(4):30. doi: 10.1007/s11912-019-0783-5.

18. Cao R, Wang L, Wang H, Xia L, Erdjument-Bromage H, Tempst P, Jones RS, Zhang Y. Role of histone $\mathrm{H} 3$ lysine 27 methylation in Polycomb-group silencing. Science. 2002;298(5595):1039-1043. doi: 10.1126/science.1076997.

19. Li Z, Li M, Wang D, Hou P, Chen X, Chu S, Chai D, Zheng J, Bai J. Post-translational modifications of EZH2 in cancer. Cell Biosci. 2020;10(1):143. doi: 10.1186/s13578-020-00505-0.

20. Nakagawa S, Okabe H, Ouchi M, Tokunaga R, Umezaki N, Higashi T, Kaida T, Arima K, Kitano Y, Kuroki H, Mima K, Nitta H, Imai K, Hashimoto D, Yamashita Y, Chikamoto A, Baba H. Enhancer of zeste homolog 2 (EZH2) regulates tumor angiogenesis and predicts recurrence and prognosis of intrahepatic cholangiocarcinoma. HPB (Oxford). 2018;20(10):939-948. doi: 10.1016/j.hpb.2018.03.018.

21. Li Z, Hou P, Fan D, Dong M, Ma M, Li H, Yao R, Li Y, Wang G, Geng P, Mihretab A, Liu D, 

the invasion and metastasis of breast cancer. Cell Death Differ. 2017;24(1):59-71. doi: 10.1038/cdd.2016.95.

22. Chen L, Jia J, Zang Y, Li J, Wan B. MicroRNA-101 regulates autophagy, proliferation and apoptosis via targeting EZH2 in laryngeal squamous cell carcinoma. Neoplasma. 2019;66(4):507-515. doi: 10.4149/neo_2018_180811N611.

23. Zheng S, Houseman EA, Morrison Z, Wrensch MR, Patoka JS, Ramos C, Haas-Kogan DA, McBride S, Marsit CJ, Christensen BC, Nelson HH, Stokoe D, Wiemels JL, Chang SM, Prados MD, Tihan T, Vandenberg SR, Kelsey KT, Berger MS, Wiencke JK. DNA hypermethylation profiles associated with glioma subtypes and EZH2 and IGFBP2 mRNA expression. Neuro-oncology. 2011;13(3):280-289. doi: 10.1093/neuonc/noq190.

24. Zhang Y, Yu X, Chen L, Zhang Z, Feng S. EZH2 overexpression is associated with poor prognosis in patients with glioma. Oncotarget. 2017;8(1):565-573. doi: 10.18632/oncotarget.13478.

25. Yu T, Wang Y, Hu Q, Wu W, Wu Y, Wei W, Han D, You Y, Lin N, Liu N. The EZH2 inhibitor GSK343 suppresses cancer stem-like phenotypes and reverses mesenchymal transition in glioma cells. Oncotarget. 2017;8(58):98348-98359. doi: 10.18632/oncotarget.21311.

26. Xu T, Wang W, Ma P, Shuai Y, Zhao K, Wang Y, Li W, Xia R, Chen W, Zhang E, Shu Y. Upregulation of the long noncoding RNAFOXD2-AS1 promotes carcinogenesis by epigenetically silencing EphB3 through EZH2 and LSD1, and predicts poor prognosis in gastric cancer. Oncogene. 2018;37(36):5020-5036. doi: 10.1038/s41388-018-0308-y. 

10.1016/j.yexcr.2019.04.016.

28. Ren Z, Hu Y, Li G, Kang Y, Liu Y, Zhao H. HIF-1 $\alpha$ induced long noncoding RNAFOXD2-AS1 promotes the osteosarcoma through repressing p21. Biomed Pharmacother. 2019;117:109104. doi: 10.1016/j.biopha.2019.109104.

29. Ni W, Xia Y, Bi Y, Wen F, Hu D, Luo L. FoxD2-AS1 promotes glioma progression by regulating miR-185-5P/HMGA2 axis and PI3K/AKT signaling pathway. Aging (Albany NY). 2019;11(5):1427-1439. doi: 10.18632/aging.101843.

B. Long noncoding RNA FOXD2-AS1 promotes glioma malignancy and tumorigenesis via targeting miR-185-5p/CCND2 axis. J Cell Biochem. 2019;120(6):9324-9336. doi: 10.1002/jcb.28208.

31. Shangguan W, Lv X, Tian N. FoxD2-AS1 is a prognostic factor in glioma and promotes temozolomide resistance in a O6-methylguanine-DNAmethyltransferase-dependent manner. Korean J Physiol Pharmacol. 2019;23(6):475-482. doi: 10.4196/kjpp.2019.23.6.475. Journal of Cancer. 2019;10(24):6074-6087. doi: 10.7150/jca.33421. glioma. Anti-Tumor Pharmacy. 2019;9(2):308-311. doi: 10.3969/j.issn.2095-1264. Postoperative Radiotherapy plus Chemotherapy on Patients with High Grade Glioma. 
Chinese Journal of Medical Guide. 2018;20(2):87 - 92. doi:

35. Li HB. Clinical Observation of Shenghe power assisted three-dimensional conformal radiotherapy and concurrent chemotherapy for the treatment of old glioma postoperative patients. Modern Journal of Integrated Traditional Chinese and Western Medicine. 2018;27(21):2339-2342. doi: 10.3969/j.issn.1008-8849.

36. Xie CJ, Zhang ZQ, Tan QJ, Huang T, Li C, Shan WG. Clinical Observation of Shenqi Fuzheng Injection on Postoperative Malignant Glioma. Journal of New Chinese Medicine. 2018;50(08):148-150. doi: 10.13457/j.cnki.jncm.2018.08.044.

37. Ma X, Yu M, Hao C, Yang W. Shikonin induces tumor apoptosis in glioma cells via permeability. J Ethnopharmacol. 2020;263:113059. doi: 10.1016/j.jep.2020.113059.

38. Li P, Chen S, Xiangfei D, Mao C, Wu CH, Shih JC. PAMs inhibits monoamine oxidase a

39. You S, He X, Wang M, Mao L, Zhang L. Tanshinone IIA Suppresses Glioma Cell Proliferation, Migration and Invasion Both in vitro and in vivo Partially Through miR-16-5p/Talin-1 (TLN1) Axis. Cancer Manag Res. 2020;12:11309-11320. doi: 10.2147/CMAR.S256347.

40. Wang J, Huang F, Bai Z, Chi B, Wu J, Chen X. Curcumol inhibits growth andinduces apoptosis of colorectal cancer lovo cell line via igf-1r and p38 mapk pathway. Int J MolSci. 2015;16(8):19851-19867. doi: 10.3390/ijms160819851. 
41. Ning N, Liu S, Liu X, Tian Z, Jiang Y, Yu N, Tan B, Feng H, Feng X, Zou L. Curcumol inhibits the proliferation and metastasis of melanoma via the miR-152-3p/PI3K/AKT and ERK/NF-kappaB signaling pathways. J Cancer. 2020;11(7):1679-1692. doi: 10.7150/jca.38624.

42. Zang S, Tang Q, Dong F, Liu H, Li L, Guo F, Pan X, Lin H, Zeng W, Cai Z, Zhong Q, Zang N, Zang L. Curcumol inhibits the proliferation of gastric adenocarcinoma MGC-803 cells via downregulation of IDH1. Oncol Rep. 2017;38(6):3583-3591. doi: 10.3892/or.2017.6028.

43. Janaki RM, Divyapriya K, Kartik KS, Rajesh YBRD. Drug-induced modifications and modulations of microRNAs and long non-coding RNAs for future therapy against Glioblastoma Multiforme. Gene. 2020;723:144126. doi: 10.1016/j.gene.2019.144126.

44. Zhou L, Wei E, Zhou B, Bi G, Gao L, Zhang T, Huang J, Wei Y, Ge B. Anti-proliferative benefit of curcumol on human bladder cancer cells via inactivating EZH2 effector. Biomedicine \& Pharmacotherapy. 2018;104:798-805. doi: 10.1016/j.biopha.2018.05.101.

\section{Figure legends}

Fig.1. Curcumol inhibited the proliferation, metastasis and promoted apoptosis in glioma cells. (A) MTT assay was performed to determine the effect of curcumol on the proliferation of glioma cells. (B) Transwell migration and (C) invasion assays were performed to determine the effect of curcumol on the metastasis of glioma cells. (D) Glioma cells were treated with curcumol for $48 \mathrm{~h}$ and analyzed by flow cytometry after AnnexinV-FITC/PI staining. Data were represented as means $\pm \mathrm{SD}$ from at least of three independent experiments. ${ }^{*} p<$ $0.05, * * p<0.01$ when compared with the untreated control group.

Fig. 2. Curcumol treatment inhibited the self-renewal ability of glioma cells. (A) CD133, Nanog, 
and DAPI staining shown by immunostaining in neurosphere from glioma stem-like cells with or without $40 \mu \mathrm{g} / \mathrm{mL}$ curcumol. Scale bar=200 $\mu \mathrm{m}$. (B) Neurosphere formation capacity of glioma stem-like cells was determined. Curcumol treatment reduced neurosphere formation of glioma stem-like cells, Scale bar=150 $\mu \mathrm{m}$. (C) The percentage of CD133+ cells was analyzed by FCM. (D) The expressions of stem cell marker genes in glioma cells were detected by western blotting analysis. ${ }^{*} p<0.05,{ }^{* *} p<0.01$ when compared with the untreated control group.

Fig. 3. Curcumol sensitized TMZ-resistant glioma cells. (A) Scheme for establishment of TMZ-resistant U251 cells. Briefly, the parental U251 cells were cultured in DMEM medium containing increasing concentrations of TMZ until they acquired resistance to TMZ. (B) MTT assay was performed to compare cell viability against TMZ in resistant cells (U251/TMZ) and the parental U251 cells. (C) Isobologram analysis the effects of curcumol combined with TMZ on cell proliferation of U251/TMZ cells. (D) Colony formation assay was used to assess clonogenicity of U251/TMZ cells following treatment with different concentrations of curcumol and/or TMZ for 14 days. (E) MS-PCR analysis of the MGMT promoter methylation status and the western blot analysis of MGMT protein level in U251/TMZ cells following treatment with different concentrations of curcumol and/or TMZ. The density of each band was quantified using imaging analysis and the relative band density values were calculated as the ratio of methylated MGMT to that of methylated plus un-methylated MGMT. MGMT unmethylated: $92 \mathrm{bp}$; MGMT methylated: $81 \mathrm{bp}$; U: unmethylated; M: methylated; MM: molecular marker. ${ }^{*} P<0.05,{ }^{* *} P<0.01$.

Fig 4. FoxD2-As1 expression was up-regulated in the glioma cells and down-regulated after 
curcumol treatment. (A) The expression of FoxD2-As1 was detected in the normal human astrocytes (NHAs) and glioma cell lines. (B) The expression of FoxD2-As1 was detected in glioma cells treatment with curcumol for 24,48 , and 72 h. $* P<0.05, * * P<0.01$.

Fig. 5. Over-expression of FoxD2-As1 reduced the anti-proliferation, anti-metastasis, and pro-apoptosis effects of curcumol in glioma cells. (A) U251 and A172 cells were transfected with pcDNA3.1-FOXD2-AS1 or empty vector, followed by treatment with 40 $\mu \mathrm{g} / \mathrm{mL}$ curcumol for $48 \mathrm{~h}$. (B) MTT assay was performed to determine the viability of each group cells after treated with curcumol for 48h. (C) Transwell migration and (D) invasion assays were performed to examine the metastasis of each group cells after treated with curcumol for $48 \mathrm{~h}$. (E) Flow cytometry analysis was performed to examine the apoptotic rate of each group cells after treated with curcumol for $48 \mathrm{~h}$. Data were represented as means $\pm \mathrm{SD}$ from at least of three independent experiments. ${ }^{*} p<0.05, * *$ $p<0.01$ when compared with the untreated control group.

Fig. 6. Over-expression of FoxD2-As1 abolished the inhibitory effect of curcumol on the self-renewal ability of glioma cells. (A) Immunostaining showed the expression of CD133 and Nanog on the membrane of glioma stem-like cells in each group, Scale bar $=200 \mu \mathrm{m}$. (B) Neurosphere formation assay showed that FoxD2-As1 upregulation promoted curcumol reduced neurosphere formation capacity of glioma cells in stem-like conditions, Scale bar $=150 \mu \mathrm{m}$. (C) Flow cytometry showed that curcumol reduced CD133+ cells were increased by upregulation of FoxD2-As1. (D) Western blot analysis showed that forced expression of FoxD2-As1 reversed curcumol-mediated decrease of CD133, Nestin, Nanog, and SOX-2 protein expression. * $p<0.05, * * p<0.01$. 
682

683

684

685

686

687

688

689

690

691

692

693

694

695

696

697

698

699

Fig. 7. Over-expression of FoxD2-As1 abolished the effect of curcumol on TMZ-resistance of glioma cells. (A) MTT assay showed that FoxD2-As1 overexpression increased the cell viability compared with control and empty vector groups. (B) Colony formation assay showed that clonogenicity of U251/TMZ cells reduced by curcumol and TMZ co-treatment was reversed by FoxD2-As1 overexpression. (C) FoxD2-As1 overexpression significantly reduced the ratio of methylated MGMT promoter DNA and promoted MGMT protein level in U251/TMZ cells. ${ }^{*} P<0.05, * * P<0.01$. FoxD2-As1 overexpression VS. control group; ${ }^{\#} P<0.05,{ }^{\#} P<0.01$. FoxD2-As1 overexpression VS. empty vector group.

Fig.8. Curcumol inhibited the activation of EZH2 through downregulation of FoxD2-As1. (A) ChIP results showed curcumol reduced the binding within the EZH2 and H3K27me3 with p21, EphB3, and CDKN1B. (B) DNA pull down results showed curcumol reduced the binding within the EZH2 and H3K27me3 with p21, EphB3, and CDKN1B. (C) curcumol inhibited the mRNA expression level of p21, EphB3, and CDKN1B in U251 and A172 cells. (D) curcumol inhibited the expression of EZH2 in U251 and A172 cells. $* P<0.05$, ** $P<$ 0.01. FoxD2-As1 overexpression VS. control group; ${ }^{\#} P<0.05$, ${ }^{\#} P<0.01$. FoxD2-As1 overexpression VS. empty vector group.

Fig.9. Curcumol inhibited malignant biological behaviors and TMZ-resistance of glioma cells in vivo. (A) Representative $\mathrm{BALB} / \mathrm{c}$ nude mice and tumors from each treatment group. Curcumol treatment significantly reduced 251/TMZ cells-derived tumor volume and weight. (B) Tumor biopsies from curcumol group had lower ability to generate spheres as compared to those of control and DMSO groups. (C) The CD133+ cell percentage in tumor sample of curcumol group was obviously lower than that in control and DMSO groups. (D) The cells 

from tumor sample of curcumol group showed low tolerance to TMZ. (E) Western blot results showed that curcumol reduced the level of CD133, MMP2, and MGMT in tumors. (F) IHC results showed that curcumol reduced the level of Ki67, CD133, MMP2, and MGMT in tumors. $* P<0.05, * * P<0.01$.

Fig. 10. A schema diagrasm displaying the inhibitory effects of curcumol on glioma tumorigenesis and TMZ-resistance. Based on the findings of the present, FOXD2-AS1 might be a molecular target of curcumol treatment and that curcumol performs its functions in glioma cells by repressing FOXD2-AS1-induced EZH2 activity. 


\section{Figures}

A

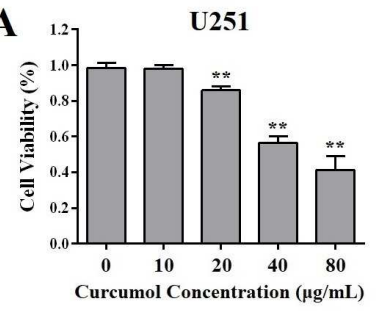

B

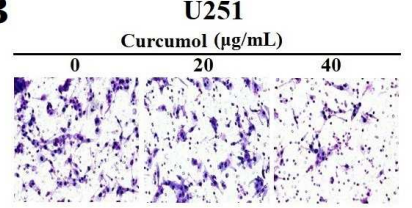

C

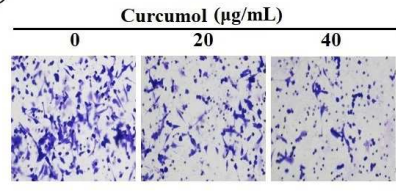

A172

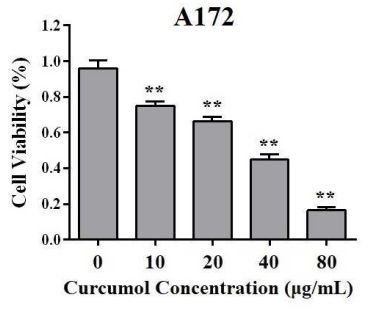

A172

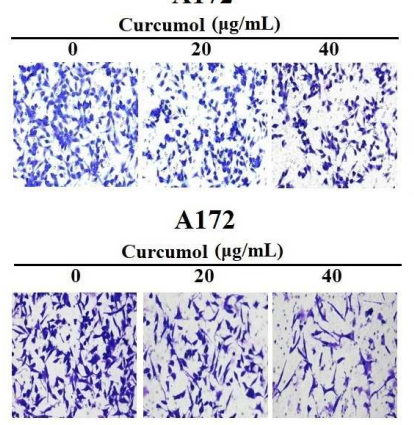

D
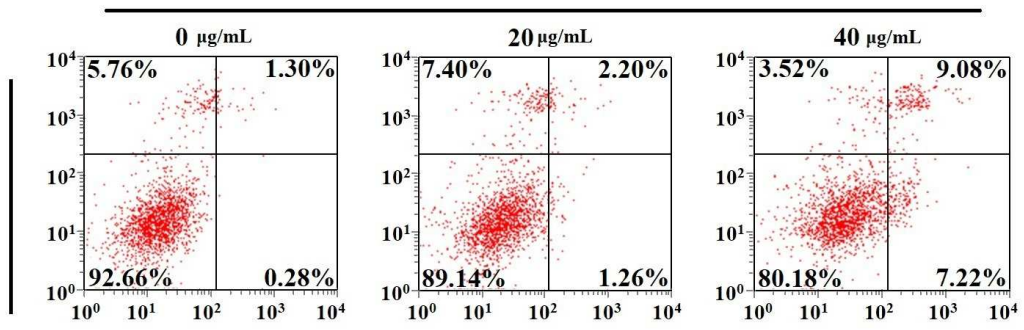

A172

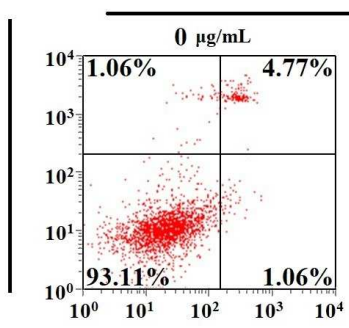

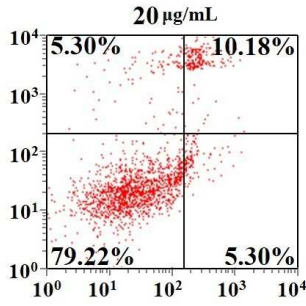

Annexin V-FITC

\section{Figure 1}

Curcumol inhibited the proliferation, metastasis and promoted apoptosis in glioma cells. (A) MTT assay was performed to determine the effect of curcumol on the proliferation of glioma cells. (B) Transwell migration and $(\mathrm{C})$ invasion assays were performed to determine the effect of curcumol on the metastasis of glioma cells. (D) Glioma cells were treated with curcumol for $48 \mathrm{~h}$ and analyzed by flow cytometry after AnnexinV-FITC/PI staining. Data were represented as means \pm SD from at least of three independent experiments. ${ }^{\star} p<0.05,{ }^{\star \star} p<0.01$ when compared with the untreated control group. 
A

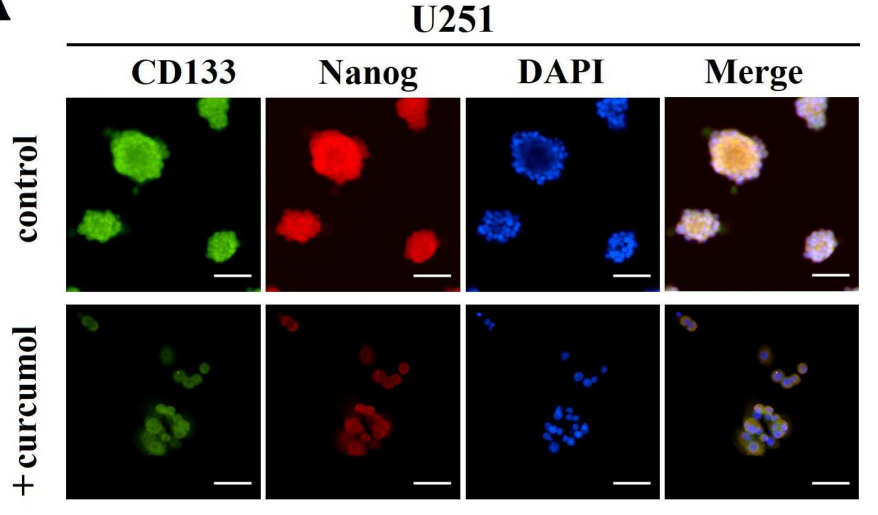

A172

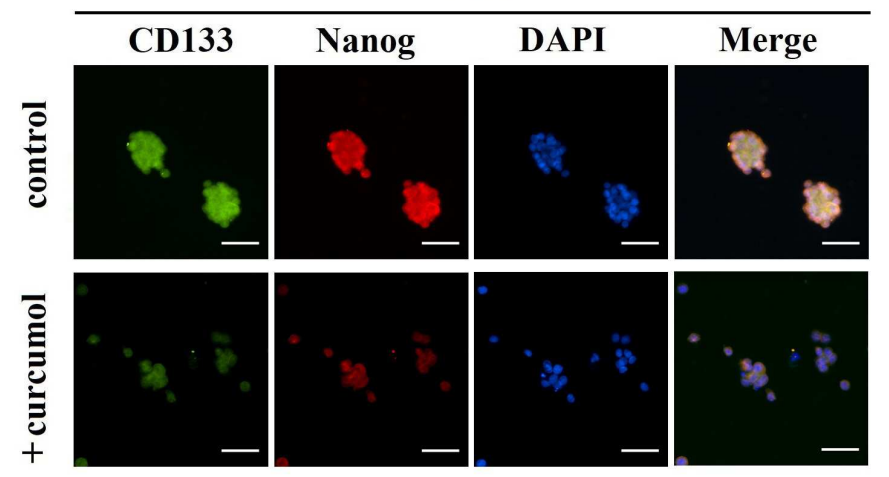

B

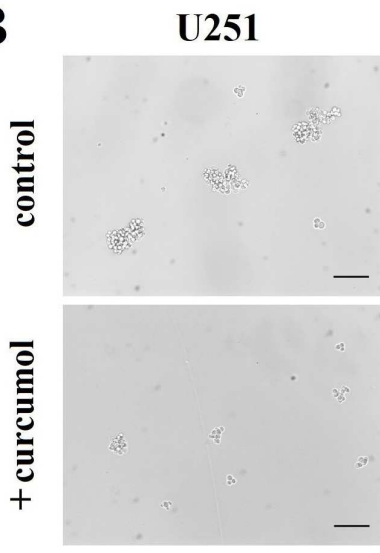

C
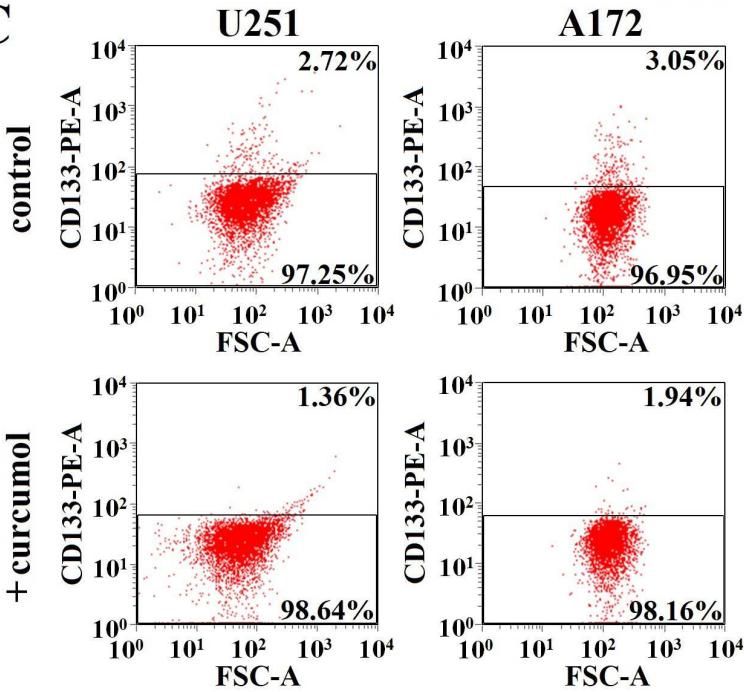

A172
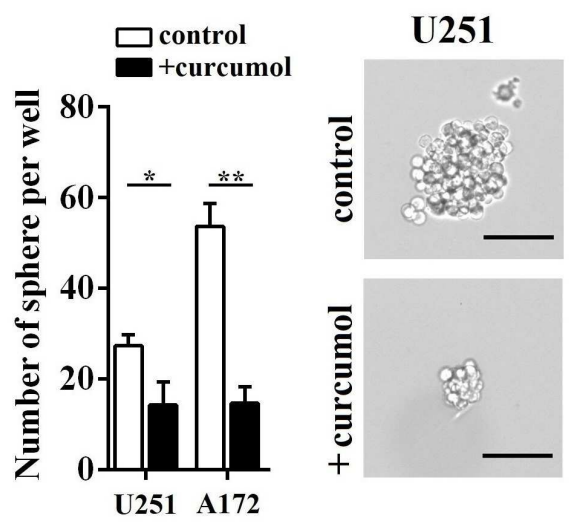

A172

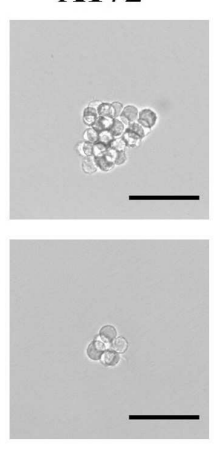

D

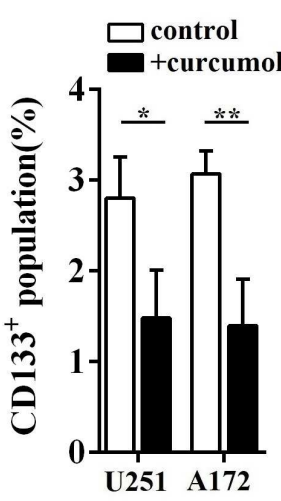

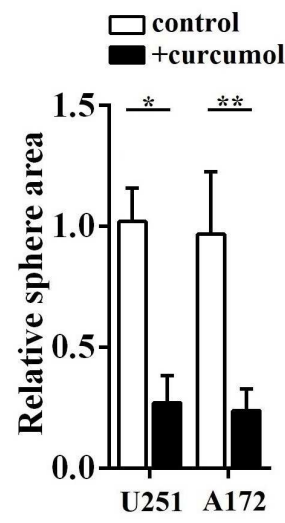

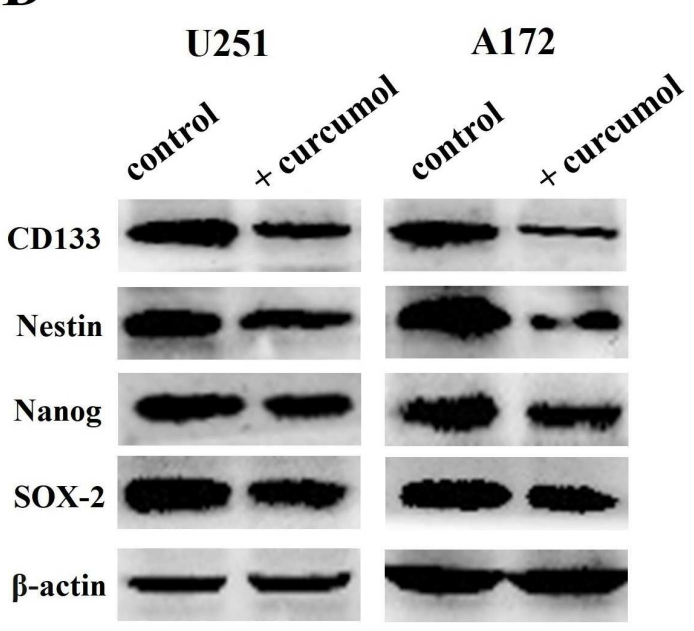

Figure 2

Curcumol treatment inhibited the self-renewal ability of glioma cells. (A) CD133, Nanog, and DAPI staining shown by immunostaining in neurosphere from glioma stem-like cells with or without $40 \mu \mathrm{g} / \mathrm{mL}$ curcumol. Scale bar $=200 \mu \mathrm{m}$. (B) Neurosphere formation capacity of glioma stem-like cells was determined. Curcumol treatment reduced neurosphere formation of glioma stem-like cells, Scale bar=150 $\mu \mathrm{m}$. (C) The percentage of CD133+ cells was analyzed by FCM. (D) The expressions of stem cell marker genes in glioma cells were detected by western blotting analysis. ${ }^{*}<0.05$, $* * p<0.01$ when compared with the untreated control group. 
A

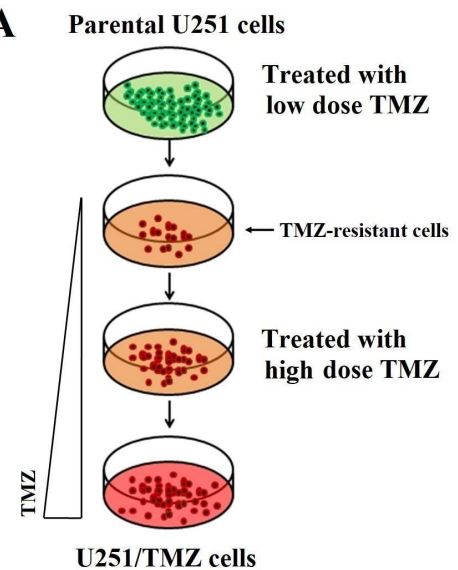

B

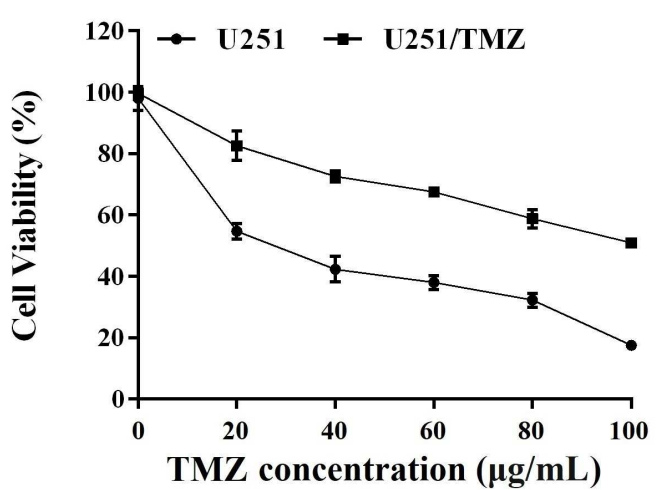

C

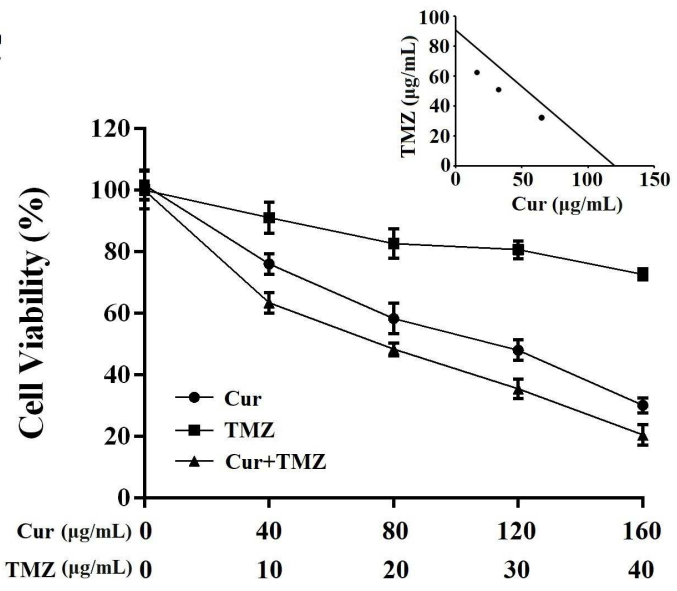

D

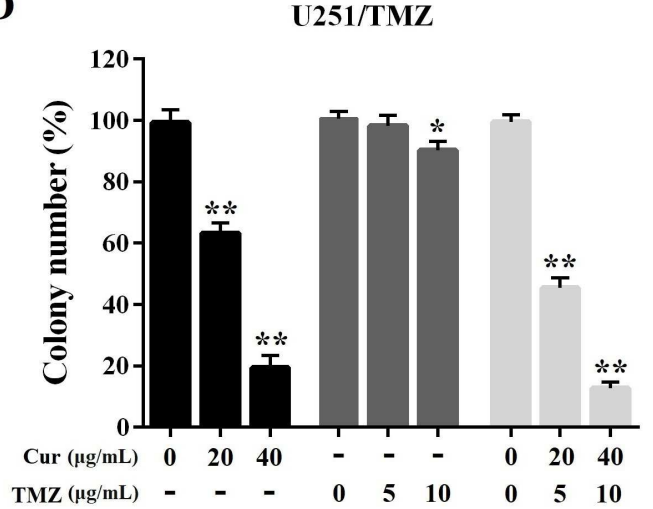

$\mathbf{E}$
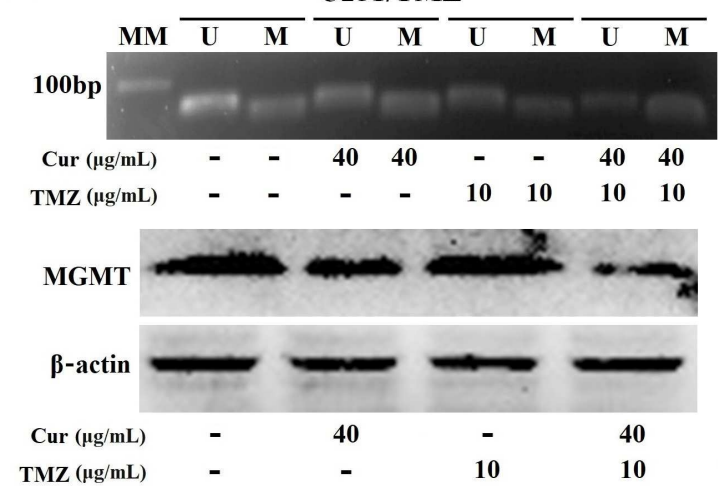

Figure 3

Curcumol sensitized TMZ-resistant glioma cells. (A) Scheme for establishment of TMZ-resistant U251 cells. Briefly, the parental U251 cells were cultured in DMEM medium containing increasing concentrations of TMZ until they acquired resistance to TMZ. (B) MTT assay was performed to compare cell viability against TMZ in resistant cells (U251/TMZ) and the parental U251 cells. (C) Isobologram analysis the effects of curcumol combined with TMZ on cell proliferation of U251/TMZ cells. (D) Colony formation assay was used to assess clonogenicity of U251/TMZ cells following treatment with different concentrations of curcumol and/or TMZ for 14 days. (E) MS-PCR analysis of the MGMT promoter methylation status and the western blot analysis of MGMT protein level in U251/TMZ cells following treatment with different concentrations of curcumol and/or TMZ. The density of each band was quantified using imaging analysis and the relative band density values were calculated as the ratio of methylated MGMT to that of methylated plus un-methylated MGMT. MGMT unmethylated: 92 bp; MGMT methylated: 8I bp; U: unmethylated; M: methylated; MM: molecular marker. ${ }^{*} \mathrm{P}<0.05,{ }^{\star \star} \mathrm{P}<0.01$. 

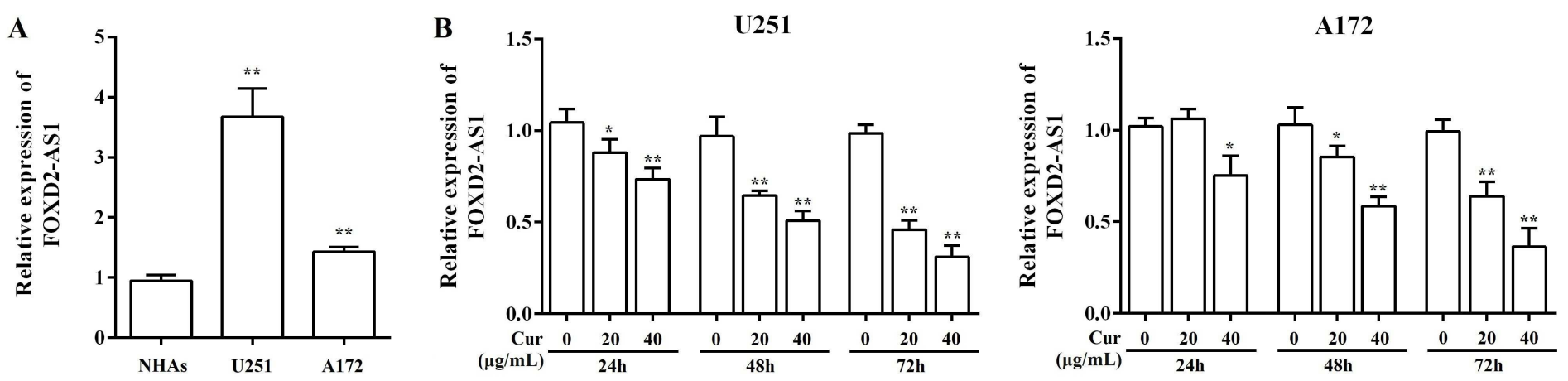

Figure 4

FoxD2-As1 expression was up-regulated in the glioma cells and down-regulated after curcumol treatment. (A) The expression of FoxD2-As1 was detected in the normal human astrocytes (NHAs) and glioma cell lines. (B) The expression of FoxD2-As1 was detected in glioma cells treatment with curcumol for 24, 48, and 72 h. ${ }^{*} \mathrm{P}<0.05, * * \mathrm{P}<0.01$. 
A

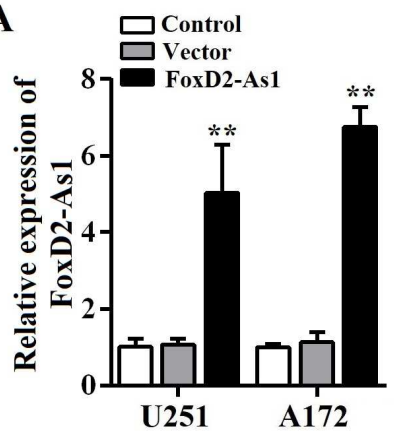

B

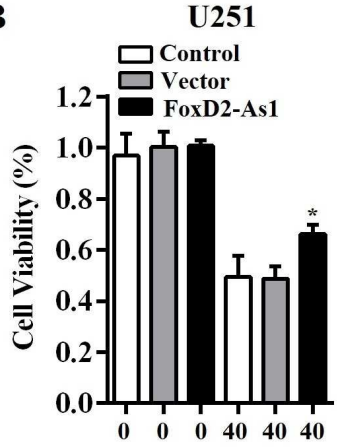

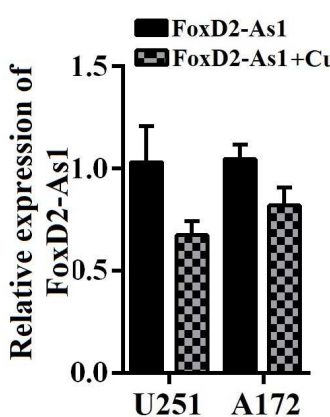

A172

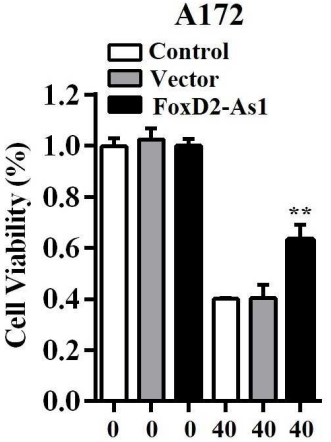

C

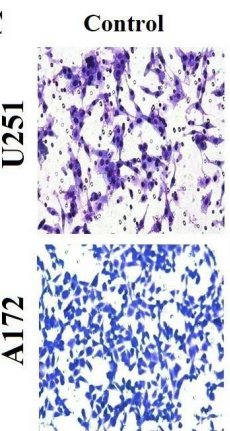

Control + Cur

Vector + Cur FoxD2-As1 + Cur

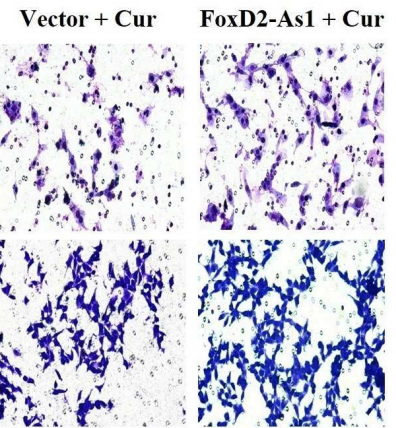

D

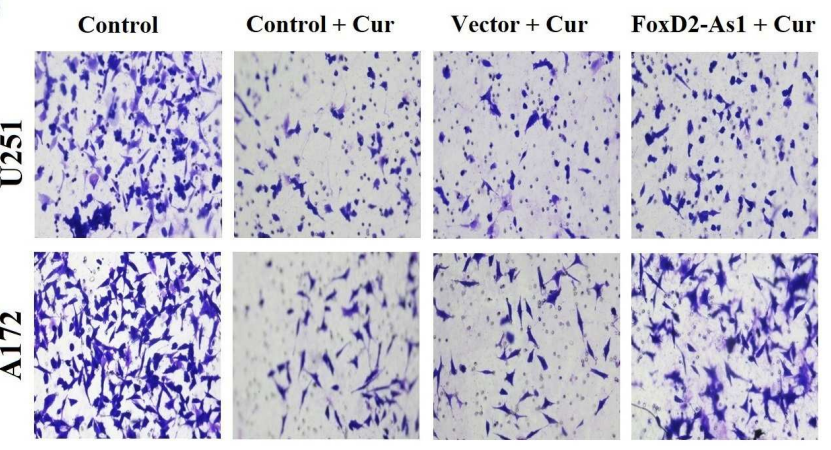

Curcumol Concentration $(\mu \mathrm{g} / \mathrm{mL})$ Curcumol Concentration $(\mu \mathrm{g} / \mathrm{mL})$

$\mathbf{E}$
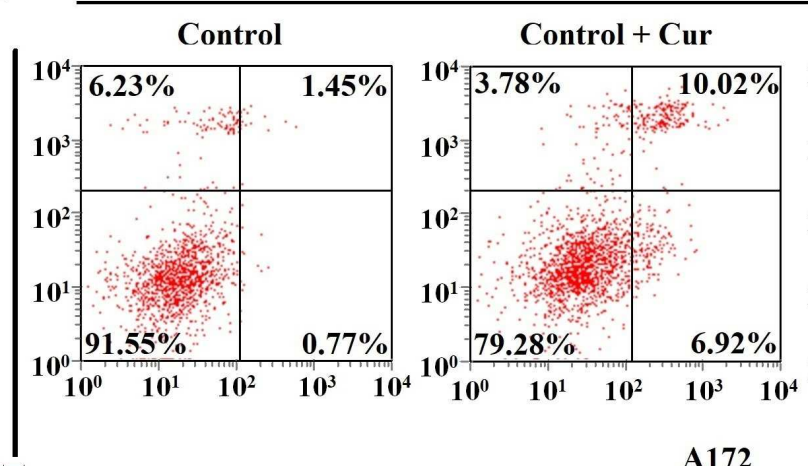

$\mathrm{U} 251$

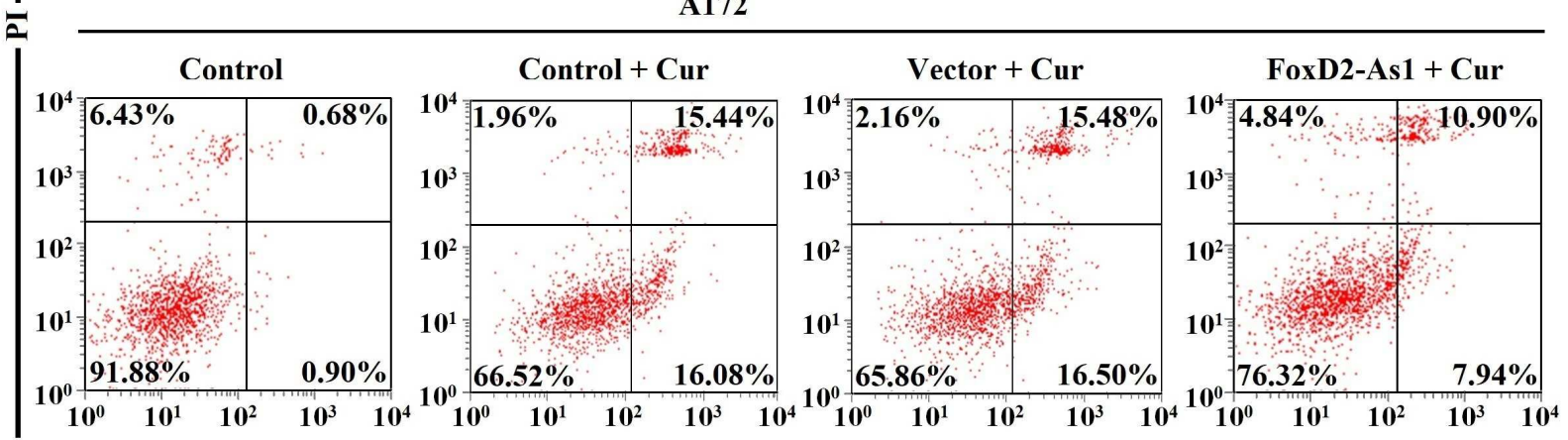

Annexin V-FITC

\section{Figure 5}

Over-expression of FoxD2-As1 reduced the anti-proliferation, anti-metastasis, and pro-apoptosis effects of curcumol in glioma cells. (A) U251 and A172 cells were transfected with pcDNA3.1-FOXD2-AS1 or empty vector, followed by treatment with $40 \mu \mathrm{g} / \mathrm{mL}$ curcumol for $48 \mathrm{~h}$. (B) MTT assay was performed to determine the viability of each group cells after treated with curcumol for $48 \mathrm{~h}$. (C) Transwell migration and (D) invasion assays were performed to examine the metastasis of each group cells after treated with 
curcumol for 48h. (E) Flow cytometry analysis was performed to examine the apoptotic rate of each group cells after treated with curcumol for $48 \mathrm{~h}$. Data were represented as means \pm SD from at least of three independent experiments. * $p<0.05$, ** $p<0.01$ when compared with the untreated control group.

A

72
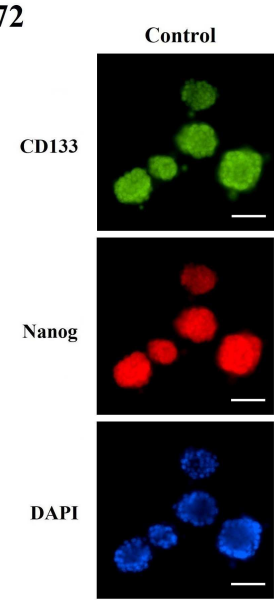

Merge

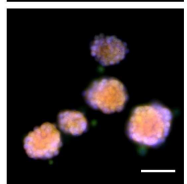

U251

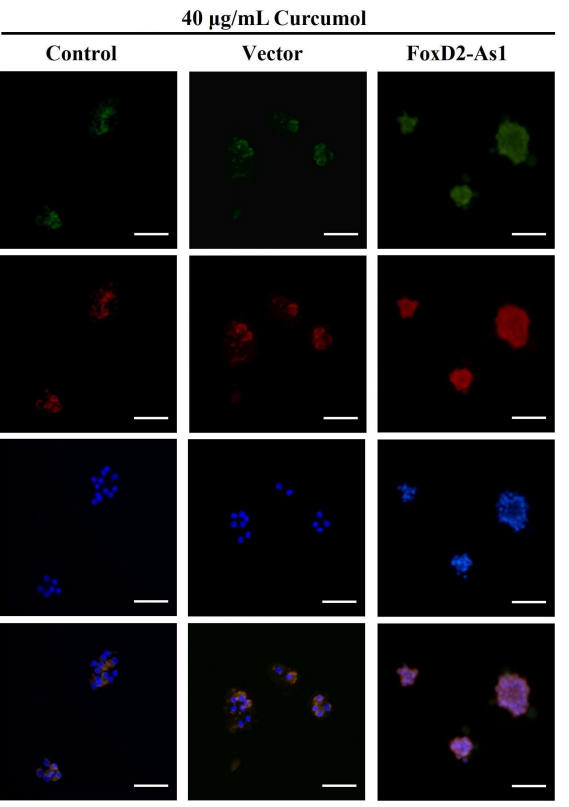

A172

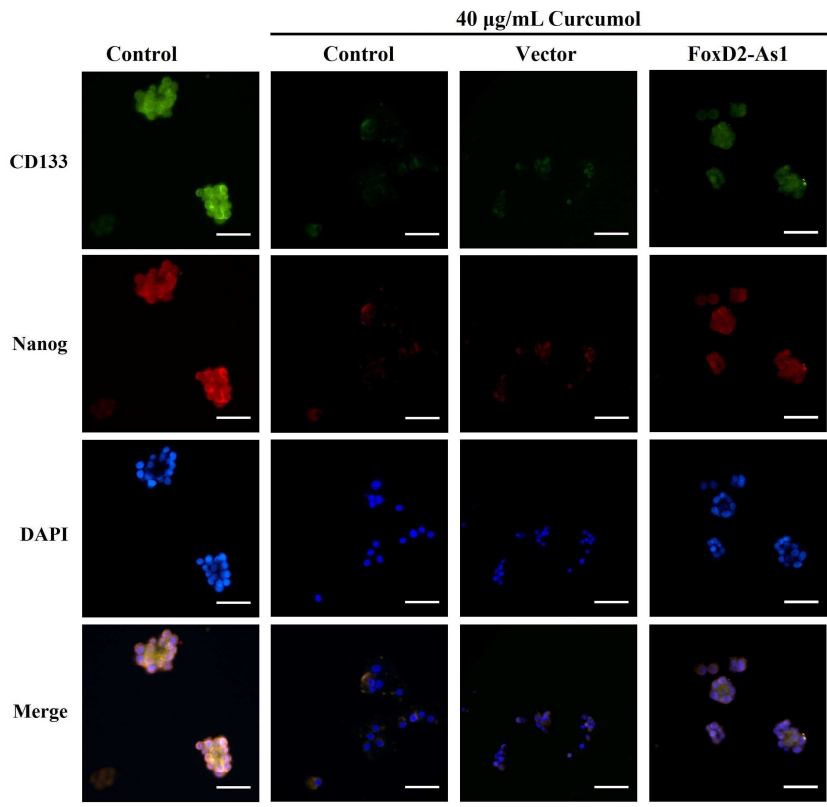

B
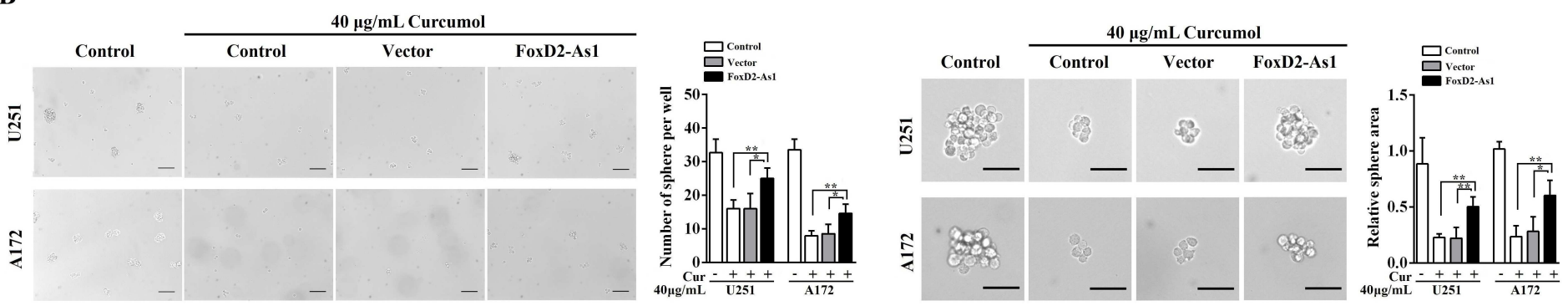

C

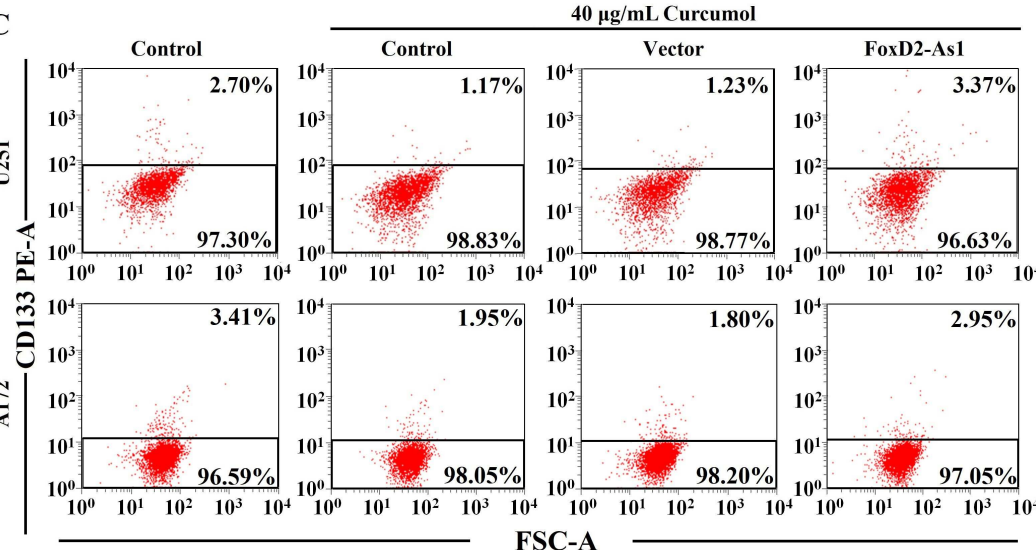

D

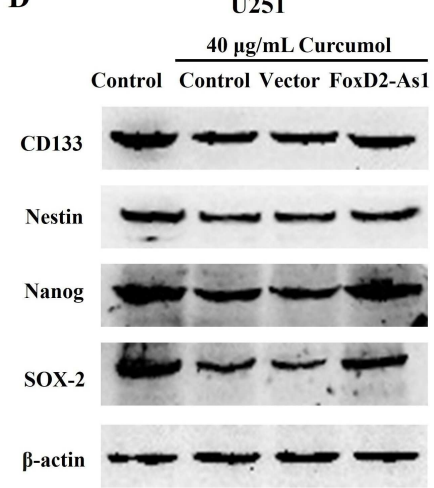

A172

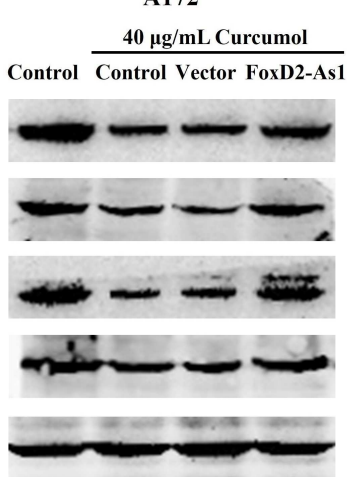

Figure 6

Over-expression of FoxD2-As1 abolished the inhibitory effect of curcumol on the self-renewal ability of glioma cells. (A) Immunostaining showed the expression of CD133 and Nanog on the membrane of glioma stem-like cells in each group, Scale bar $=200 \mu \mathrm{m}$. (B) Neurosphere formation assay showed that FoxD2-As1 upregulation promoted curcumol reduced neurosphere formation capacity of glioma cells in stem-like conditions, Scale bar $=150 \mu \mathrm{m}$. (C) Flow cytometry showed that curcumol reduced CD133+ cells 
were increased by upregulation of FoxD2-As1. (D) Western blot analysis showed that forced expression of FoxD2-As1 reversed curcumol-mediated decrease of CD133, Nestin, Nanog, and SOX-2 protein expression. ${ }^{*} p<0.05, * * p<0.01$.

A

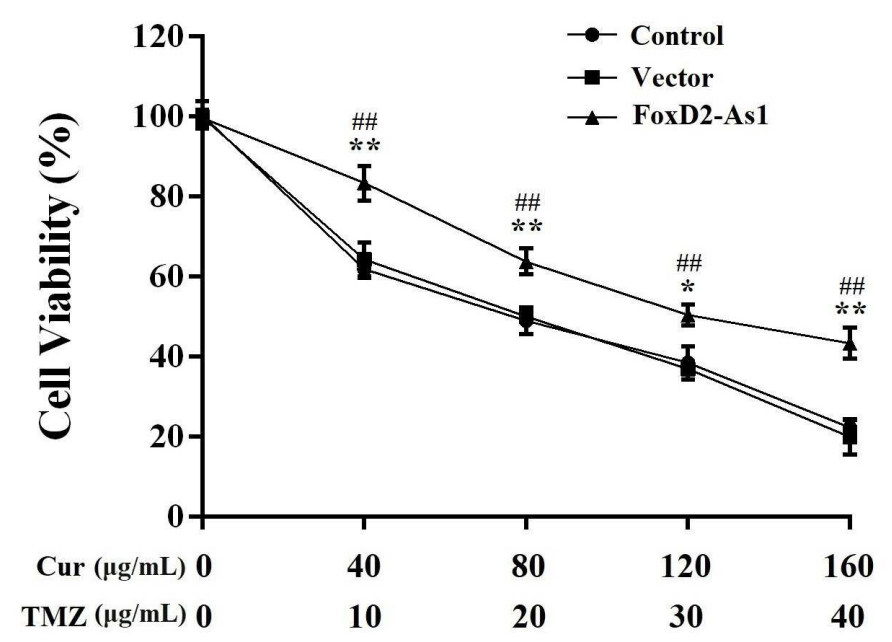

C

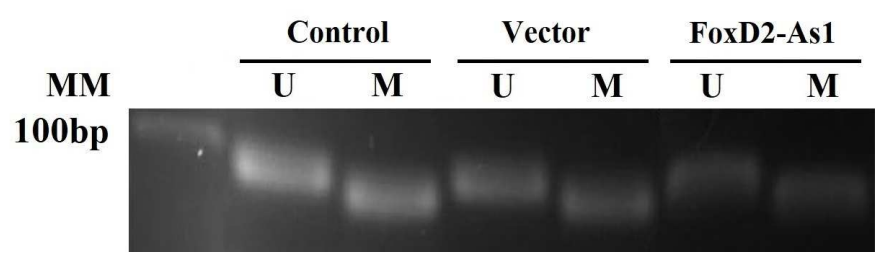

Control Vector FoxD2-As1

MGMT

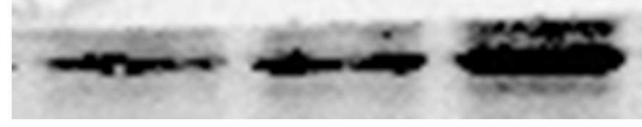

$\beta$-actin
U251/TMZ

40
B
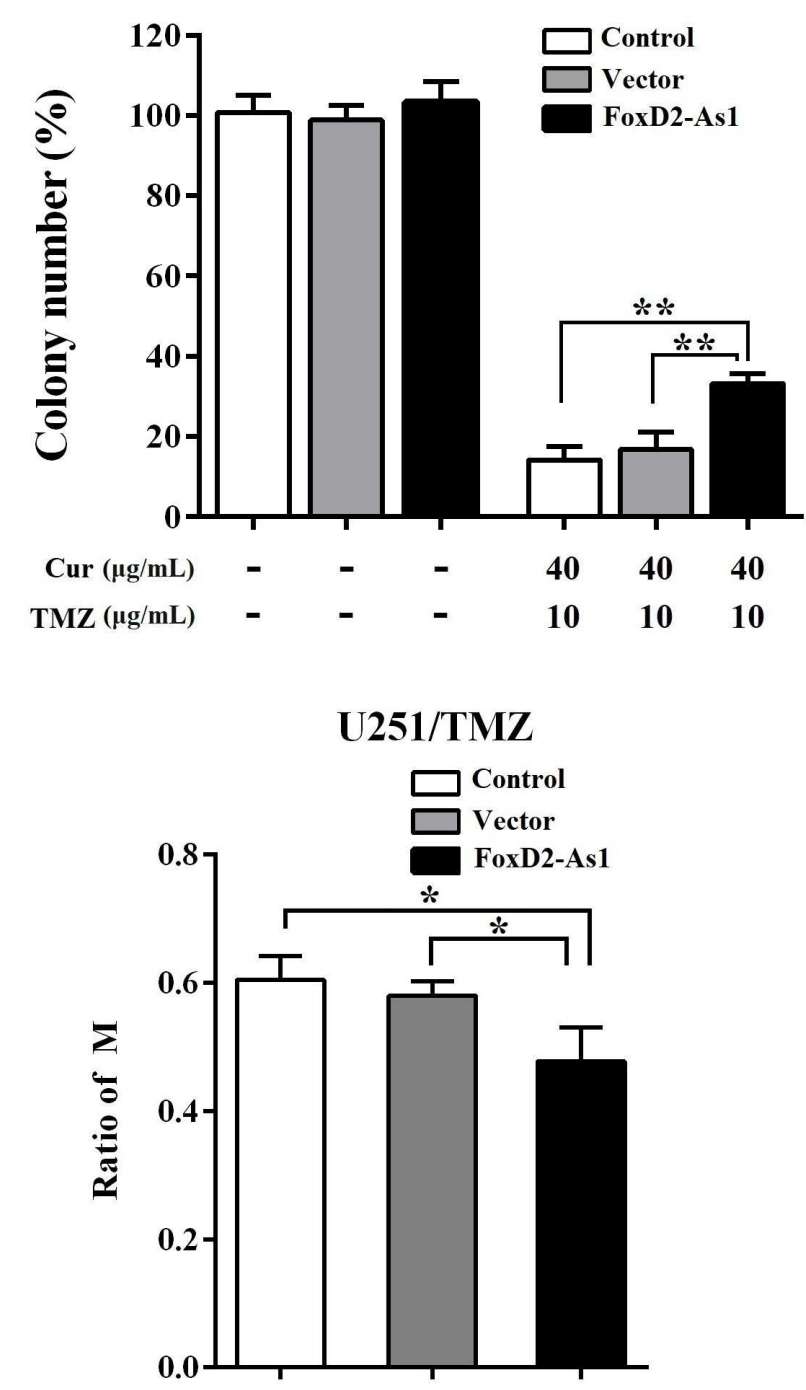

Figure 7

Over-expression of FoxD2-As1 abolished the effect of curcumol on TMZ-resistance of glioma cells. (A) MTT assay showed that FoxD2-As1 overexpression increased the cell viability compared with control and empty vector groups. (B) Colony formation assay showed that clonogenicity of U251/TMZ cells reduced by curcumol and TMZ co-treatment was reversed by FoxD2-As1 overexpression. (C) FoxD2-As1 overexpression significantly reduced the ratio of methylated MGMT promoter DNA and promoted MGMT protein level in U251/TMZ cells. ${ }^{*} \mathrm{P}<0.05,{ }^{\star \star} \mathrm{P}<0.01$. FoxD2-As1 overexpression VS. control group; \#P $<$ 0.05 , \#\#P $<0.01$. FoxD2-As1 overexpression VS. empty vector group. 
A

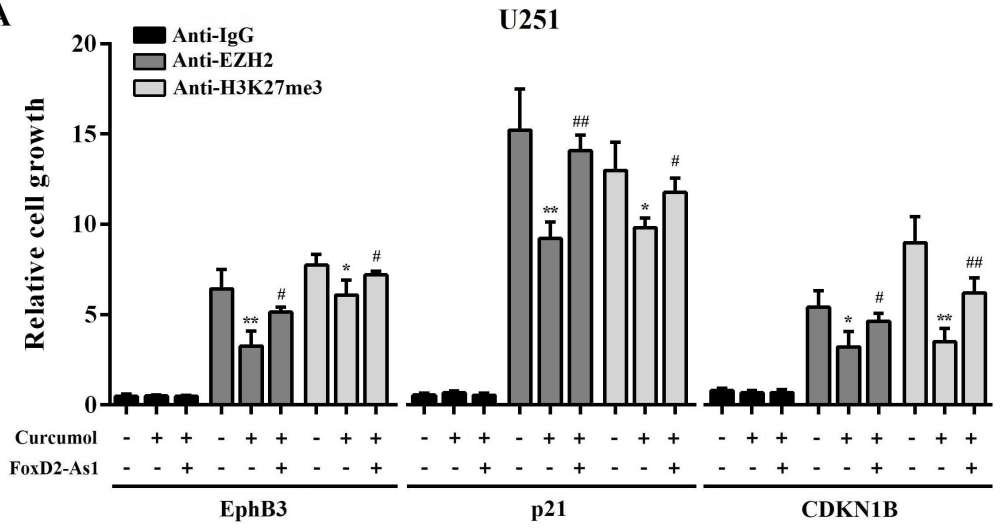

C

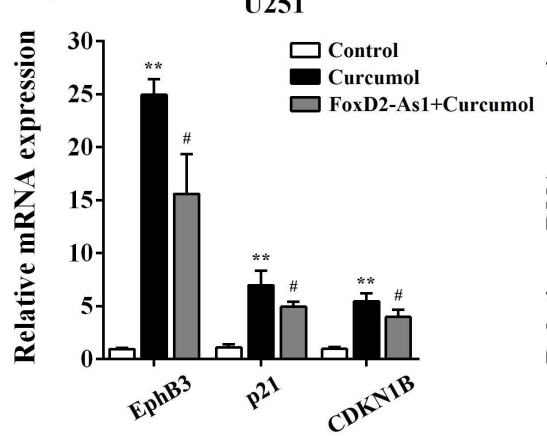

A172
B

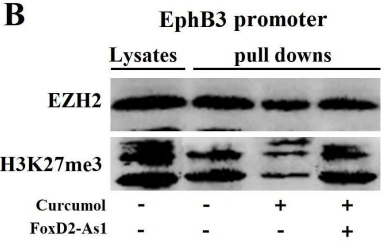

p21 promoter

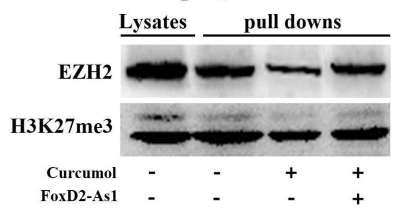

D

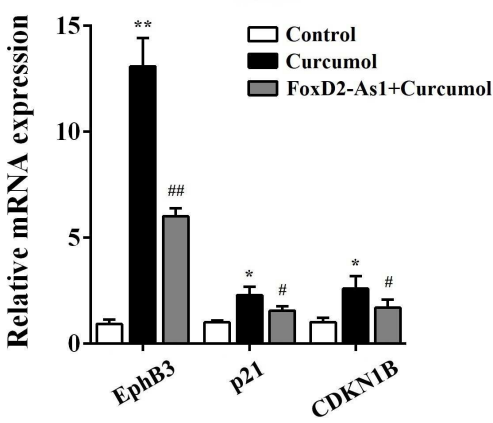

U251

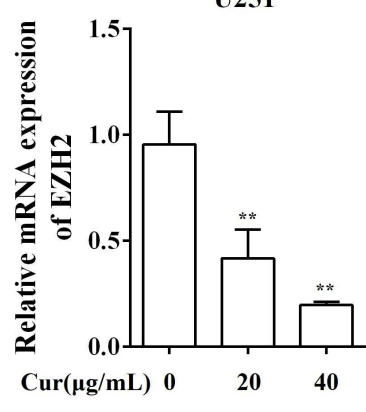

A172

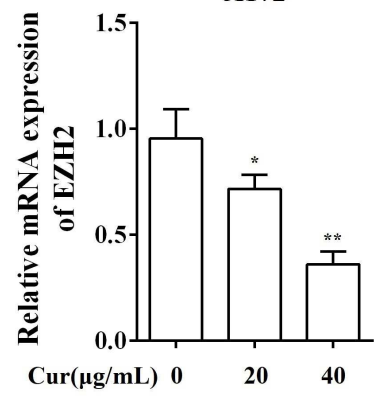

Figure 8

Curcumol inhibited the activation of EZH2 through downregulation of FoxD2-As1. (A) ChIP results showed curcumol reduced the binding within the EZH2 and H3K27me3 with p21, EphB3, and CDKN1B. (B) DNA pull down results showed curcumol reduced the binding within the EZH2 and H3K27me3 with p21, EphB3, and CDKN1B. (C) curcumol inhibited the mRNA expression level of p21, EphB3, and CDKN1B in U251 and A172 cells. (D) curcumol inhibited the expression of EZH2 in U251 and A172 cells. *P $<0.05$, $\star \star P<0.01$. FoxD2-As1 overexpression VS. control group; \#P $<0.05$, \#\#P $<0.01$. FoxD2-As1 overexpression VS. empty vector group. 
A
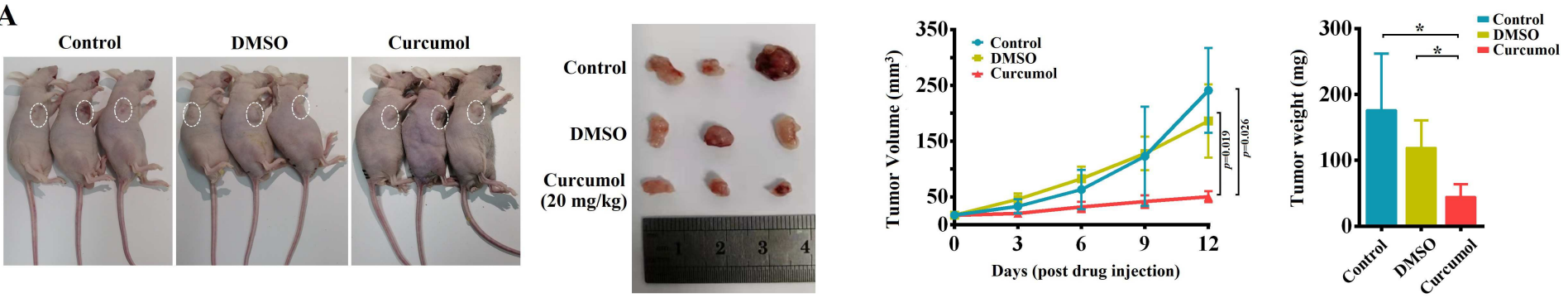

B
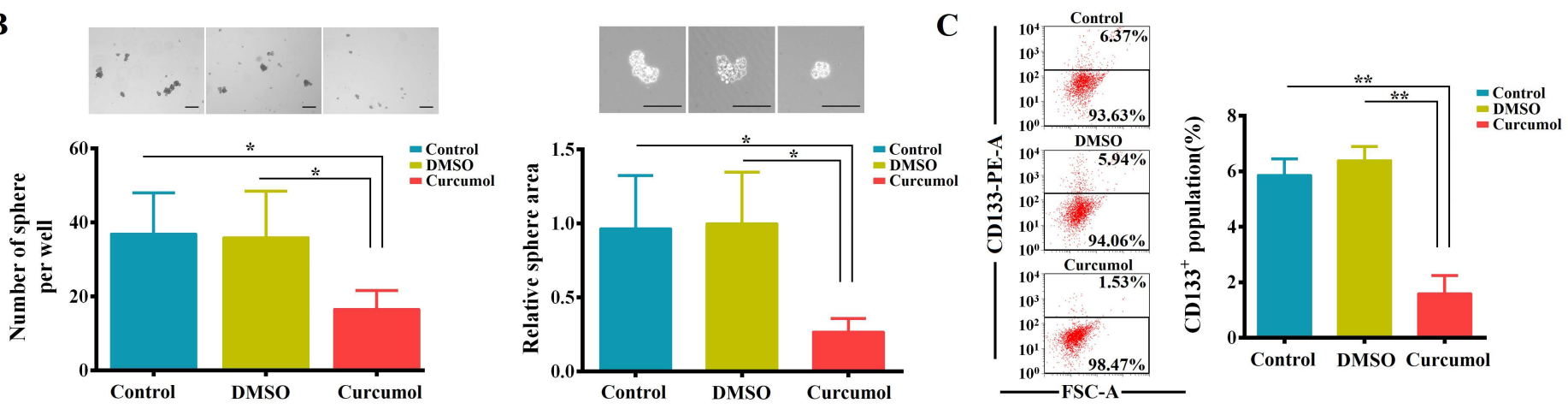

D

$\mathbf{E}$
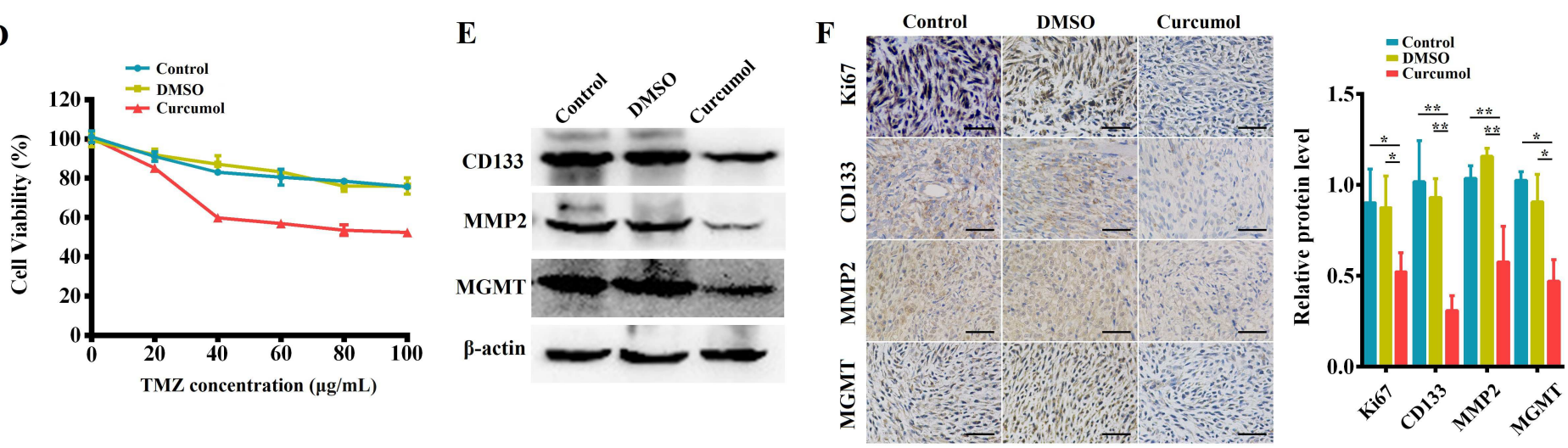

Figure 9

Curcumol inhibited malignant biological behaviors and TMZ-resistance of glioma cells in vivo. (A) Representative BALB/c nude mice and tumors from each treatment group. Curcumol treatment significantly reduced 251/TMZ cells-derived tumor volume and weight. (B) Tumor biopsies from curcumol group had lower ability to generate spheres as compared to those of control and DMSO groups. (C) The CD133+ cell percentage in tumor sample of curcumol group was obviously lower than that in control and DMSO groups. (D) The cells from tumor sample of curcumol group showed low tolerance to TMZ. (E) Western blot results showed that curcumol reduced the level of CD133, MMP2, and MGMT in tumors. (F) IHC results showed that curcumol reduced the level of Ki67, CD133, MMP2, and MGMT in tumors. ${ }^{*} \mathrm{P}<0.05, * * \mathrm{P}<0.01$. 


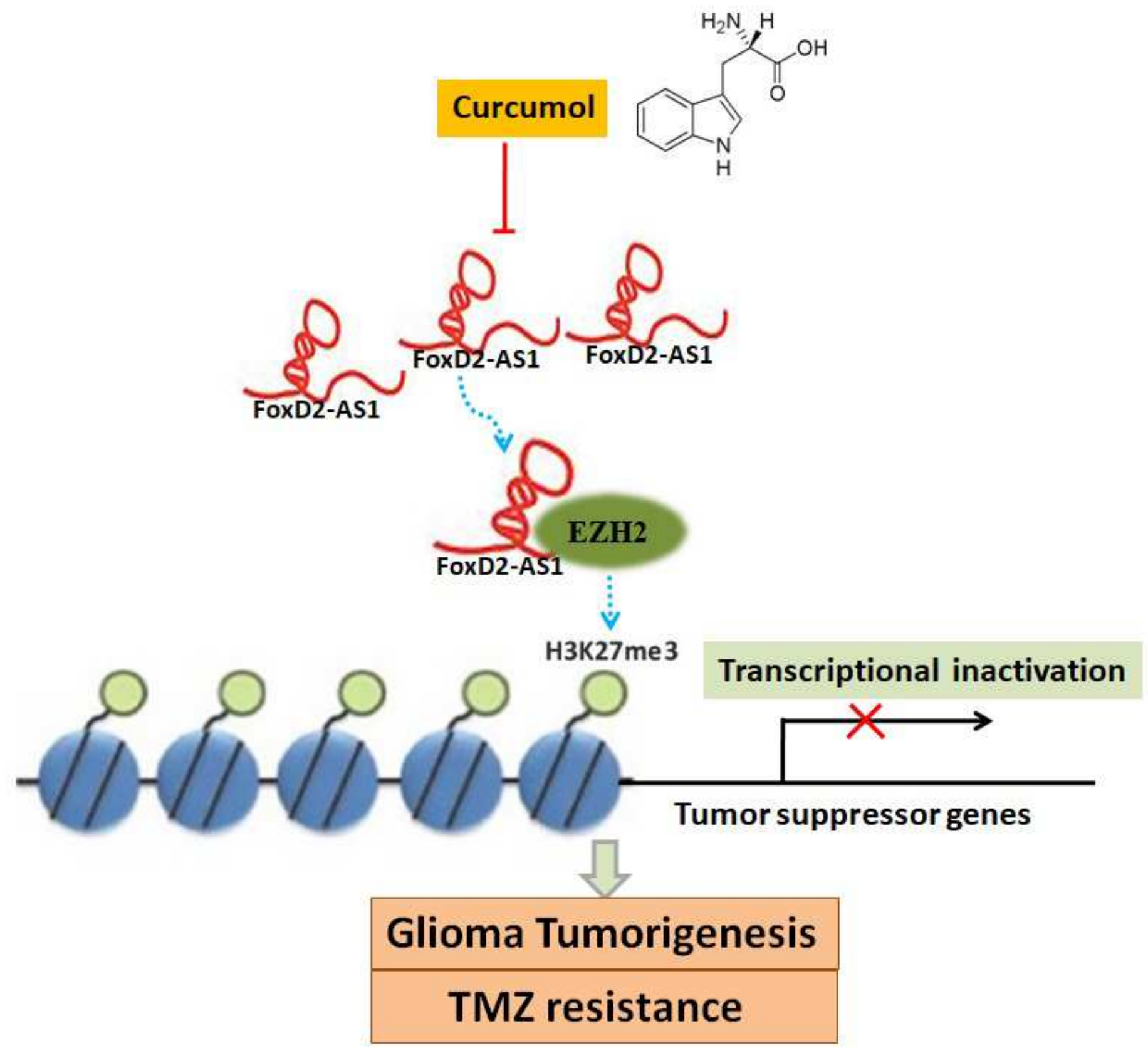

Figure 10

A schema diagrasm displaying the inhibitory effects of curcumol on glioma tumorigenesis and TMZresistance. Based on the findings of the present, FOXD2-AS1 might be a molecular target of curcumol treatment and that curcumol performs its functions in glioma cells by repressing FOXD2-AS1-induced EZH2 activity. 